\title{
Congruences on Menger algebras
}

\author{
Wieslaw A. Dudek and Valentin S. Trokhimenko
}

May 11, 2018

\begin{abstract}
We discuss some types of congruences on Menger algebras of rank $n$, which are generalizations of the principal left and right congruences on semigroups. We also study congruences admitting various types of cancellations and describe their relationship with strong subsets.
\end{abstract}

\section{Preliminaries}

Let $A$ be a nonempty set. Consider the family $\Phi$ of mappings $f: A^{n} \rightarrow A$ closed under the $(n+1)$-ary composition $f\left[g_{1} \ldots g_{n}\right]$ defined by:

$$
f\left[g_{1} \ldots g_{n}\right]\left(x_{1}, \ldots, x_{n}\right)=f\left(g_{1}\left(x_{1}, \ldots, x_{n}\right), \ldots, g_{n}\left(x_{1}, \ldots, x_{n}\right)\right) .
$$

Such defined an $(n+1)$-ary composition is called the Menger superposition of $n$-place functions (cf. [3, 4]) and satisfies the so-called superassociative law:

$$
f\left[g_{1} \ldots g_{n}\right]\left[h_{1} \ldots h_{n}\right]=f\left[g_{1}\left[h_{1} \ldots h_{n}\right] \ldots g_{n}\left[h_{1} \ldots h_{n}\right]\right],
$$

where $f, g_{i}, h_{i} \in \Phi, i=1, \ldots, n$.

Obtained algebra $(\Phi, \mathcal{O})$, where $\mathcal{O}:\left(f, g_{1}, \ldots, g_{n}\right) \mapsto f\left[g_{1} \ldots g_{n}\right]$ is a Menger algebra of n-place functions. Their abstract analog $(G, o)$, i.e., a nonempty set $G$ with an $(n+1)$-ary operation $o:\left(f, g_{1}, \ldots, g_{n}\right) \mapsto f\left[g_{1} \ldots g_{n}\right]$ satisfying (1) is called a Menger algebra $(G, o)$ of rank $n$.

Let $(G, o)$ be a Menger algebra of rank $n, e_{1}, \ldots, e_{n}$ - pairwise different elements that not belong to the set $G, \bar{e}=\left(e_{1}, \ldots, e_{n}\right)$ and $B=G^{n} \cup\{\bar{e}\}$. In addition, we assume that $g\left[e_{1} \ldots e_{n}\right]=g, e_{i}\left[g_{1} \ldots g_{n}\right]=g_{i}$ and $e_{i}\left[e_{1} \ldots e_{n}\right]=e_{i}$ for all $g, g_{1}, \ldots, g_{n} \in G$ and each $i=1, \ldots, n$. Elements $e_{1}, \ldots, e_{n}$ corresponds to the projectors $I_{1}, \ldots, I_{n}$ defined by $I_{i}\left(x_{1}, \ldots, x_{n}\right)=x_{i}$.

Since the $(n+1)$-ary operation $o$ is superassociative, the set $B$ with a binary operation:

$$
\bar{x} * \bar{y}=\left(x_{1}, \ldots, x_{n}\right) *\left(y_{1}, \ldots, y_{n}\right)=\left(x_{1}\left[y_{1} \ldots y_{n}\right], \ldots, x_{n}\left[y_{1} \ldots y_{n}\right]\right)
$$

is a semigroup with the identity $\bar{e}$. Obviously $\left(G^{n}, *\right)$ is a subsemigroup of $(B, *)$. 
Let us consider the set $T_{n}(G)$ of all expressions, called polynomials, in the alphabet $G \cup\{[], x\}$, where the square brackets and $x$ do not belong to $G$, defined as follows:

(a) $x \in T_{n}(G)$,

(b) if $i \in\{1, \ldots, n\}, \quad a, b_{1}, \ldots, b_{i-1}, b_{i+1}, \ldots, b_{n} \in G, \quad t \in T_{n}(G)$, then $a\left[b_{1} \ldots b_{i-1} t b_{i+1}, \ldots b_{n}\right] \in T_{n}(G)$,

(c) $T_{n}(G)$ contains precisely those polynomials which are defined according to $(a)$ and $(b)$.

Every polynomial $t \in T_{n}(G)$ defines on $(G, o)$ an elementary translation $t: x \mapsto t(x)$. The polynomial and the elementary translation defined by it will be noted by the same letter. Obviously, $t_{1} \circ t_{2} \in T_{n}(G)$ for any $t_{1}, t_{2} \in T_{n}(G)$, where $\left(t_{1} \circ t_{2}\right)(x)=t_{1}\left(t_{2}(x)\right)$. The identity map on the set $G$ belongs to $T_{n}(G)$ also.

A subset $H$ of a Menger algebra $(G, o)$ of rank $n$ is called

- a normal v-complex if for all $g_{1}, g_{2} \in G, t \in T_{n}(G)$

$$
g_{1} \in H \wedge g_{2} \in H \wedge t\left(g_{1}\right) \in H \longrightarrow t\left(g_{2}\right) \in H,
$$

- a normal $l$-complex if for all $g_{1}, g_{2} \in G$ and $\bar{x} \in B$

$$
g_{1} \in H \wedge g_{2} \in H \wedge g_{1}[\bar{x}] \in H \longrightarrow g_{2}[\bar{x}] \in H,
$$

- a normal bicomplex if for any $g_{1}, g_{2} \in G, t \in T_{n}(G), \bar{x} \in B$

$$
g_{1} \in H \wedge g_{2} \in H \wedge t\left(g_{1}[\bar{x}]\right) \in H \longrightarrow t\left(g_{2}[\bar{x}]\right) \in H,
$$

- an l-ideal if for all $x, h_{1}, \ldots, h_{n} \in G$

$$
\left(h_{1}, \ldots, h_{n}\right) \in G^{n} \backslash(G \backslash H)^{n} \longrightarrow x\left[h_{1} \ldots h_{n}\right] \in H,
$$

- an i-ideal $(1 \leqslant i \leqslant n)$, if for all $h, u \in G, \bar{w} \in G^{n}$

$$
h \in H \longrightarrow u\left[\left.\bar{w}\right|_{i} h\right] \in H,
$$

where $u\left[\left.\bar{w}\right|_{i} h\right]$ denotes $u\left[w_{1} \ldots w_{i-1} h w_{i+1} \ldots w_{n}\right]$,

- an s-ideal if for all $h, x_{1}, \ldots, x_{n} \in G$

$$
h \in H \longrightarrow h\left[x_{1} \ldots x_{n}\right] \in H,
$$

- an sl-ideal if $H$ is both $s$-ideal and $l$-ideal.

It is clear that $H$ is an $l$-ideal if and only if it is an $i$-ideal for all $i=1, \ldots, n$.

A binary relation $\rho$ defined on a Menger algebra $(G, o)$ of rank $n$ is called 
- stable if for all $x, y, x_{i}, y_{i} \in G, i=1, \ldots, n$

$$
(x, y),\left(x_{1}, y_{1}\right), \ldots,\left(x_{n}, y_{n}\right) \in \rho \longrightarrow\left(x\left[x_{1} \ldots x_{n}\right], y\left[y_{1} \ldots y_{n}\right]\right) \in \rho
$$

- l-regular if for any $x, y, z_{i} \in G, i=1, \ldots, n$

$$
(x, y) \in \rho \longrightarrow\left(x\left[z_{1} \ldots z_{n}\right], y\left[z_{1} \ldots z_{n}\right]\right) \in \rho,
$$

- $v$-regular if for all $x_{i}, y_{i}, z \in G, i=1, \ldots, n$

$$
\left(x_{1}, y_{1}\right), \ldots,\left(x_{n}, y_{n}\right) \in \rho \longrightarrow\left(z\left[x_{1} \ldots x_{n}\right], z\left[y_{1} \ldots y_{n}\right]\right) \in \rho
$$

- $i$-regular if for any $u, x, y \in G, \bar{w} \in G^{n}$

$$
(x, y) \in \rho \longrightarrow\left(u\left[\left.\bar{w}\right|_{i} x\right], u\left[\left.\bar{w}\right|_{i} y\right]\right) \in \rho,
$$

- l-cancellative if for all $x, y \in G,\left(z_{1}, \ldots, z_{n}\right) \in G^{n}$

$$
\left(x\left[z_{1} \ldots z_{n}\right], y\left[z_{1} \ldots z_{n}\right]\right) \in \rho \longrightarrow(x, y) \in \rho
$$

- $v$-cancellative if for all $x, y, u \in G, \bar{w} \in G^{n}, i=1, \ldots, n$

$$
\left(u\left[\left.\bar{w}\right|_{i} x\right], u\left[\left.\bar{w}\right|_{i} y\right]\right) \in \rho \longrightarrow(x, y) \in \rho,
$$

- $l v$-cancellative if it is both $l$-cancellative and $v$-cancellative.

A reflexive and transitive binary relation is $v$-regular if and only if it is $i$ regular for all $i=1, \ldots, n$. A binary relation is stable if and only if it is $l$-regular and $v$-regular (cf. 3]). A stable equivalence is a congruence. By a $v$-congruence (l-congruence) we mean an equivalence relation which is $v$-regular ( $l$-regular, respectively). It is easy to see that a relation $\rho \subset G \times G$ is $v$-cancellative if and only if

$$
(t(x), t(y)) \in \rho \longrightarrow(x, y) \in \rho
$$

holds for all $x, y \in G$ and each translation $t \in T_{n}(G)$.

The above terminology is inspired by the terminology used in semigroups. A Menger algebra of rank $n=1$ is a semigroup, so, in this case, an $l$-regular relation is left regular, and a $v$-regular (vector regular) relation is right regular in the classical sense.

In this paper we describe principal $v$-congruences and principal $l$-congruences on a Menger algebra of rank $n$. Obtained results are similar. The main scheme of proofs also is similar, but the concept of strong subsets (used in the proofs) is different in any case. Thus, these proofs are not analogous.

In the last section we characterize bistrong subsets of Menger algebras and their connections with congruences. 


\section{Principal $v$-congruences on Menger algebras}

Let $(G, o)$ be a Menger algebra of $\operatorname{rank} n, H$ its nonempty subset. Put

$$
\begin{gathered}
\rho_{H}=\left\{(a, t) \in G \times T_{n}(G) \mid t(a) \in H\right\}, \\
\rho_{H}\langle a\rangle=\left\{t \in T_{n}(G) \mid t(a) \in H\right\}, \\
\rho_{H}^{\circ}\langle t\rangle=\{a \in G \mid t(a) \in H\}, \\
\mathcal{R}_{H}=\left\{(a, b) \in G \times G \mid \rho_{H}\langle a\rangle=\rho_{H}\langle b\rangle\right\}, \\
\mathcal{R}_{H}^{*}=\left\{(a, b) \in G \times G \mid \rho_{H}\langle a\rangle=\rho_{H}\langle b\rangle \neq \varnothing\right\} .
\end{gathered}
$$

The relation $\mathcal{R}_{H}$ is an equivalence on $(G, o)$ but the relation $\mathcal{R}_{H}^{*}$ is only symmetric and transitive. The set $W_{H}=\left\{a \in G \mid \rho_{H}\langle a\rangle=\varnothing\right\}$ is a complement of the domain of $\mathcal{R}_{H}^{*}$. Thus, if $W_{H} \neq \varnothing$, then $W_{H}$ is the $\mathcal{R}_{H}$-class. Obviously, $\mathcal{R}_{H}^{*}=\mathcal{R}_{H} \cap\left(G \backslash W_{H}\right) \times\left(G \backslash W_{H}\right)$.

It is not difficult to see that for any $a, b \in G$ we have

$$
\begin{gathered}
(a, b) \in \mathcal{R}_{H} \longleftrightarrow\left(\forall t \in T_{n}(G)\right)(t(a) \in H \longleftrightarrow t(b) \in H), \\
a \in W_{H} \longleftrightarrow\left(\forall t \in T_{n}(G)\right)(t(a) \notin H) .
\end{gathered}
$$

Observe that $H \cap W_{H}=\varnothing$. Indeed, for $h \in H \cap W_{H}$ we have $h \in H$ and $t(h) \notin H$ for any $t \in T_{n}(G)$. This for the identity translation $t(x)=x$ gives $h \in H$ and $h \notin H$, which is impossible. So, $H \cap W_{H}=\varnothing$.

Proposition 2.1. $\mathcal{R}_{H}$ is a v-congruence.

Proof. Let $(a, b) \in \mathcal{R}_{H}$. Then, according to (5), for every $t \in T_{n}(G)$ we have $t(a) \in H \longleftrightarrow t(b) \in H$. In particular $t\left(u\left[\left.\bar{w}\right|_{i} a\right]\right) \in H \longleftrightarrow t\left(u\left[\left.\bar{w}\right|_{i} b\right]\right) \in H$ for every $u \in G, \bar{w} \in G^{n}$ and $i \in\{1, \ldots, n\}$. So, $\left(u\left[\left.\bar{w}\right|_{i} a\right], u\left[\left.\bar{w}\right|_{i} b\right]\right) \in \mathcal{R}_{H}$. Hence, the relation $\mathcal{R}_{H}$ is $i$-regular for every $i=1, \ldots, n$. Consequently, it is a $v$-congruence on $(G, o)$.

Proposition 2.2. $W_{H}$ is an l-ideal, provided that it is a nonempty set.

Proof. Let $W_{H} \neq \varnothing$ and $a \in W_{H}$. Then, according to (6), $t(a) \notin H$ for each translation $t \in T_{n}(G)$. Hence $t_{1}\left(u\left[\left.\bar{w}\right|_{i} a\right]\right) \notin H$ for $t_{1}\left(u\left[\left.\bar{w}\right|_{i} a\right]\right) \notin H$, where $t_{1} \in T_{n}(G), u \in G, \bar{w} \in G^{n}$ and $i \in\{1, \ldots, n\}$. Thus, $u\left[\left.\bar{w}\right|_{i} a\right] \in W_{H}$ for each $i=1, \ldots, n$, i.e., $W_{H}$ is an $l$-ideal of a Menger algebra $(G, o)$.

The relation $\mathcal{R}_{H}$ is called the principal v-congruence, the relation $\mathcal{R}_{H}^{*}$ - the principal partial $v$-congruence on a Menger algebra $(G, o)$. The set $W_{H}$ is called the $v$-residue of $H$.

Proposition 2.3. If a nonempty subset $H$ of a Menger algebra $(G, o)$ is its normal $v$-complex, then it is an $\mathcal{R}_{H}$-class different from $W_{H}$. 
Proof. Let $h_{1}, h_{2} \in H$. Suppose that $t\left(h_{1}\right) \in H$ for some $t \in T_{n}(G)$. Then $h_{1}, h_{2}, t\left(h_{1}\right) \in H$, which gives $t\left(h_{2}\right) \in H$ because $H$ is a normal $v$-complex. So, $t\left(h_{1}\right) \in H$ implies $t\left(h_{2}\right) \in H$. Analogously, $t\left(h_{2}\right) \in H$ implies $t\left(h_{1}\right) \in H$. Thus, according to (5), $\left(h_{1}, h_{2}\right) \in \mathcal{R}_{H}$ for all $h_{1}, h_{2} \in H$. Hence $H$ is contained in some $\mathcal{R}_{H}$-class. Denote this class by $X$. Now let $h \in H$ and $g$ be an arbitrary element of $X$. Then $(h, g) \in \mathcal{R}_{H}$, i.e., for each $t \in T_{n}(G)$ we have $t(h) \in H \longleftrightarrow t(g) \in H$. This for the identity translation gives $h \in H \longleftrightarrow g \in H$. So, $H=X$. Since $H \cap W_{H}=\varnothing$, the $\mathcal{R}_{H}$-class $H$ is different from $W_{H}$.

Proposition 2.4. Any v-congruence $\varepsilon$ on a Menger algebra $(G, o)$ has the form

$$
\varepsilon=\bigcap\left\{\mathcal{R}_{H} \mid H \in G / \varepsilon\right\},
$$

where $G / \varepsilon$ is the set of all $\varepsilon$-classes.

Proof. Let $\left(g_{1}, g_{2}\right) \in \varepsilon$. Consider an arbitrary $\varepsilon$-class $H$. If $t\left(g_{1}\right) \in H$ for some $t \in T_{n}(G)$, then $\left(t\left(g_{1}\right), t\left(g_{2}\right)\right) \in \varepsilon$ because the relation $\varepsilon$ is $i$-regular for every $i=1, \ldots, n$. So $t\left(g_{2}\right) \in H$. Analogously, $t\left(g_{2}\right) \in H$ gives $t\left(g_{1}\right) \in H$. Thus, according to (5), we have $\left(g_{1}, g_{2}\right) \in \mathcal{R}_{H}$, i.e., $\left(g_{1}, g_{2}\right) \in \varepsilon$ implies $\left(g_{1}, g_{2}\right) \in \mathcal{R}_{H}$. Hence $\varepsilon \subseteq \bigcap\left\{\mathcal{R}_{H} \mid H \in G / \varepsilon\right\}$.

Conversely, let $\left(g_{1}, g_{2}\right) \in \bigcap\left\{\mathcal{R}_{H} \mid H \in G / \varepsilon\right\}$. Then, according to (5), for all $H \in G / \varepsilon$ and $t \in T_{n}(G)$ we have

$$
t\left(g_{1}\right) \in H \longleftrightarrow t\left(g_{2}\right) \in H .
$$

From this, for the identity translation $t$ and the $\varepsilon$-class $H_{1}$ containing $g_{1}$, we obtain $g_{1} \in H_{1} \longleftrightarrow g_{2} \in H_{1}$. Thus $\left(g_{1}, g_{2}\right) \in \varepsilon$. Consequently, $\bigcap\left\{\mathcal{R}_{H} \mid H \in\right.$ $G / \varepsilon\} \subseteq \varepsilon$. This completes the proof.

Definition 2.5. A nonempty subset $H$ of a Menger algebra $(G, o)$ is called $v$-admissible if it is an equivalence class of some $v$-congruence on $(G, o)$.

Proposition 2.6. Each v-admissible subset $H$ of a Menger algebra $(G, o)$ is contained in some $\mathcal{R}_{H}$-class. Moreover, if $\rho_{H}\langle h\rangle \neq \varnothing$ for some $h \in H$, then this $\mathcal{R}_{H}$-class is different from $W_{H}$.

Proof. Let $H$ be a $v$-admissible subset of a Menger algebra $(G, o)$. Then it is some $\varepsilon$-class of a $v$-congruence $\varepsilon$. Therefore, by Proposition 2.4 we obtain $\varepsilon \subseteq \mathcal{R}_{H}$. So, $H$ is contained in some $\mathcal{R}_{H}$-class. If $\rho_{H}\langle h\rangle \neq \varnothing$ for some $h \in H$, then $h \notin W_{H}$. This means that the $\mathcal{R}_{H}$-class containing $H$ cannot be the set $W_{H}$.

Definition 2.7. A subset $H$ of a Menger algebra $(G, o)$ is called strong if

$$
\rho_{H}\langle a\rangle \cap \rho_{H}\langle b\rangle \neq \varnothing \longrightarrow \rho_{H}\langle a\rangle=\rho_{H}\langle b\rangle
$$

for all $a, b \in G$.

Theorem 2.8. For a subset $H$ of a Menger algebra $(G, o)$ the following conditions are equivalent: 
(a) $H$ is strong.

(b) For any $x, y \in G$ and $t_{1}, t_{2} \in T_{n}(G)$

$$
t_{1}(x) \in H \wedge t_{1}(y) \in H \wedge t_{2}(y) \in H \longrightarrow t_{2}(x) \in H .
$$

(c) For any $t_{1}, t_{2} \in T_{n}(G)$

$$
\rho_{H}^{\circ}\left\langle t_{1}\right\rangle \cap \rho_{H}^{\circ}\left\langle t_{2}\right\rangle \neq \varnothing \longrightarrow \rho_{H}^{\circ}\left\langle t_{1}\right\rangle=\rho_{H}^{\circ}\left\langle t_{2}\right\rangle .
$$

Proof. $(a) \longrightarrow(b)$. Let $H$ be a strong subset of a Menger algebra $(G, o)$. If $t_{1}(x), t_{1}(y), t_{2}(y) \in H$ for some $x, y \in G$ and $t_{1}, t_{2} \in T_{n}(G)$, then $t_{1} \in \rho_{H}\langle x\rangle \cap$ $\rho_{H}\langle y\rangle$. Thus $\rho_{H}\langle x\rangle \cap \rho_{H}\langle y\rangle \neq \varnothing$, so $\rho_{H}\langle x\rangle=\rho_{H}\langle y\rangle$. But $t_{2}(y) \in H$ means that $t_{2} \in \rho_{H}\langle y\rangle$. Hence $t_{2} \in \rho_{H}\langle x\rangle$, i.e., $t_{2}(x) \in H$. This proves $(b)$.

$(b) \longrightarrow(c)$. Suppose that $\rho_{H}^{\circ}\left\langle t_{1}\right\rangle \cap \rho_{H}^{\circ}\left\langle t_{2}\right\rangle \neq \varnothing$ for some $t_{1}, t_{2} \in T_{n}(G)$. Then there exists $x \in G$ such that $x \in \rho_{H}^{\circ}\left\langle t_{1}\right\rangle \cap \rho_{H}^{\circ}\left\langle t_{2}\right\rangle$, i.e., $t_{1}(x) \in H$ and $t_{2}(x) \in H$. If $y \in \rho_{H}^{\circ}\left\langle t_{1}\right\rangle$, i.e., $t_{1}(y) \in H$, then, by (8), we have $t_{2}(y) \in H$, i.e., $y \in \rho_{H}^{\circ}\left\langle t_{2}\right\rangle$. So, $\rho_{H}^{\circ}\left\langle t_{1}\right\rangle \subseteq \rho_{H}^{\circ}\left\langle t_{2}\right\rangle$. Similarly we obtain $\rho_{H}^{\circ}\left\langle t_{2}\right\rangle \subseteq \rho_{H}^{\circ}\left\langle t_{1}\right\rangle$. This proves $(c)$.

$(c) \longrightarrow(a)$. Let $\rho_{H}\langle a\rangle \cap \rho_{H}\langle b\rangle \neq \varnothing$ for some $a, b \in G$. Then $t_{1} \in \rho_{H}\langle a\rangle \cap \rho_{H}\langle b\rangle$ for some $t_{1} \in T_{n}(G)$, i.e., $t_{1}(a)$ and $t_{1}(b)$ are in $H$. Consequently, $a, b \in \rho_{H}^{\circ}\left\langle t_{1}\right\rangle$. Let $t_{2}$ is any translation from $\rho_{H}\langle a\rangle$, then $t_{2}(a) \in H$, i.e., $a \in \rho_{H}^{\circ}\left\langle t_{2}\right\rangle$. Therefore $\rho_{H}^{\circ}\left\langle t_{1}\right\rangle \cap \rho_{H}^{\circ}\left\langle t_{2}\right\rangle \neq \varnothing$ which, by (9), gives $\rho_{H}^{\circ}\left\langle t_{1}\right\rangle=\rho_{H}^{\circ}\left\langle t_{2}\right\rangle$. As $b \in \rho_{H}^{\circ}\left\langle t_{1}\right\rangle$, then, obviously, $b \in \rho_{H}^{\circ}\left\langle t_{2}\right\rangle$, i.e., $t_{2}(b) \in H$, whence $t_{2} \in \rho_{H}\langle b\rangle$. So, $\rho_{H}\langle a\rangle \subseteq$ $\rho_{H}\langle b\rangle$. The reverse inclusion follows similarly. Thus, $\rho_{H}\langle a\rangle=\rho_{H}\langle b\rangle$. So, $H$ is strong.

Remark. The empty subset of a Menger algebra is strong since for $H=\varnothing$ the premise and the conclusion of the implication (8) are false, so it is true.

Proposition 2.9. Every nonempty strong subset $H$ of a Menger algebra $(G, o)$ is an $\mathcal{R}_{H}$-class different from $W_{H}$.

Proof. Let $H \neq \varnothing$ be a strong subset of a Menger algebra $(G, o)$. Then it satisfies the condition $(b)$ of Theorem 2.8. Replacing $t_{1}$ in (8) by the identity translation we see that $H$ is a normal $v$-complex. Proposition 2.3 completes the proof.

Proposition 2.10. Let $H$ be a strong subset of a Menger algebra $(G, o)$ of rank $n$. Then all $\mathcal{R}_{H}$-classes are nonempty members of the family

$$
\mathcal{K}=\left\{\rho_{H}^{\circ}\langle t\rangle \mid t \in T_{n}(G)\right\} \cup\left\{W_{H}\right\} .
$$

Proof. Firts, we show that $\mathcal{K}$ is a partition of $G$. Obviously, $\bigcup \mathcal{K} \subseteq G$ since $W_{H} \subset G$ and $\rho_{H}^{\circ}\langle t\rangle \subset G$ for each $t \in T_{n}(G)$. Let $g$ be an arbitrary element of $G$. Then either $t(g) \notin H$ for all $t \in T_{n}(G)$, or $t(g) \in H$ for some $t \in T_{n}(G)$. In the first case $g \in W_{H}$, in the second $g \in \rho_{H}^{\circ}\langle t\rangle$ for some $t \in T_{n}(G)$. So, $G \subseteq \bigcup \mathcal{K}$. Thus, $G=\bigcup \mathcal{K}$. This means that $\mathcal{K}$ covers $G$.

If $g \in \rho_{H}^{\circ}\left\langle t_{1}\right\rangle \cap W_{H}$, where $t_{1} \in T_{n}(G)$, then $t_{1}(g) \in H$ and $t(g) \notin H$ for all $t \in T_{n}(G)$. So, $t_{1}(g) \in H$ and $t_{1}(g) \notin H$. This is impossible. Therefore 
$\rho_{H}^{\circ}\left\langle t_{1}\right\rangle \cap W_{H}=\varnothing$ for each $t_{1} \in T_{n}(G)$. Now, if $g \in \rho_{H}^{\circ}\left\langle t_{1}\right\rangle \cap \rho_{H}^{\circ}\left\langle t_{2}\right\rangle$, where $t_{1}, t_{2} \in T_{n}(G)$, then $\rho_{H}^{\circ}\left\langle t_{1}\right\rangle \cap \rho_{H}^{\circ}\left\langle t_{2}\right\rangle \neq \varnothing$. Thus $\rho_{H}^{\circ}\left\langle t_{1}\right\rangle=\rho_{H}^{\circ}\left\langle t_{2}\right\rangle$ because $H$ is a strong subset of $(G, o)$. So, if $\rho_{H}^{\circ}\left\langle t_{1}\right\rangle \neq \rho_{H}^{\circ}\left\langle t_{2}\right\rangle$, then $\rho_{H}^{\circ}\left\langle t_{1}\right\rangle \cap \rho_{H}^{\circ}\left\langle t_{2}\right\rangle=\varnothing$. Hence $\mathcal{K}$ is a partition of $G$.

Now let $(a, b) \in \mathcal{R}_{H}$, i.e., $\rho_{H}\langle a\rangle=\rho_{H}\langle b\rangle$. If $\rho_{H}\langle a\rangle=\varnothing$, then $a, b \in W_{H}$, and as it is noted above, $W_{H}$ is one of the $\mathcal{R}_{H}$-classes. If $\rho_{H}\langle a\rangle \neq \varnothing$, then there is a translation $t \in T_{n}(G)$ such that $t \in \rho_{H}\langle a\rangle=\rho_{H}\langle b\rangle$, so $t(a) \in H$ and $t(b) \in H$. Consequently, $a, b \in \rho_{H}^{\circ}\langle t\rangle$.

Conversely, if $a, b \in \rho_{H}^{\circ}\langle t\rangle$ for some $t \in T_{n}(G)$, then $t(a) \in H$ and $t(b) \in H$, so $t \in \rho_{H}\langle a\rangle \cap \rho_{H}\langle b\rangle$. Thus, $\rho_{H}\langle a\rangle \cap \rho_{H}\langle b\rangle \neq \varnothing$, hence, $\rho_{H}\langle a\rangle=\rho_{H}\langle b\rangle$, i.e., $(a, b) \in \mathcal{R}_{H}$. Therefore $\rho_{H}^{\circ}\langle t\rangle$ is the $\mathcal{R}_{H}$-class. In this way we have shown that $\mathcal{K}$ is a family of $\mathcal{R}_{H}$-classes.

Theorem 2.11. Let $H$ be a strong subset of a Menger algebra $(G, o)$. If $X \neq$ $W_{H}$ is an $\mathcal{R}_{H}$-class, then $X$ is a strong subset of $(G, o)$ such that $W_{H} \subseteq W_{X}$ and $\mathcal{R}_{H} \subseteq \mathcal{R}_{X}$. Moreover, the restriction of $\mathcal{R}_{H}$ to $G \backslash W_{X}$ coincides with $\mathcal{R}_{X}^{*}$.

Proof. Since $\mathcal{R}_{H}$-class $X$ is different from $W_{H}$, there exists $t \in T_{n}(G)$ such that $X=\rho_{H}^{\circ}\langle t\rangle$ (Proposition 2.10). Let $y, z \in G$ and $\rho_{X}\langle y\rangle \cap \rho_{X}\langle z\rangle \neq \varnothing$. Then for some translation $t_{1} \in T_{n}(G)$ we have $t_{1} \in \rho_{X}\langle y\rangle \cap \rho_{X}\langle z\rangle$. This means that $t_{1}(y)$ and $t_{1}(z)$ are in $\rho_{H}^{\circ}\langle t\rangle$. So, $t\left(t_{1}(y)\right)$ and $t\left(t_{1}(z)\right)$ are in $H$. Consequently, $\left(t \circ t_{1}\right)(y)$ and $\left(t \circ t_{1}\right)(z)$ also are in $H$, i.e., $t \circ t_{1} \in \rho_{H}\langle y\rangle$ and $t \circ t_{1} \in \rho_{H}\langle z\rangle$, whence $\rho_{H}\langle y\rangle \cap \rho_{H}\langle z\rangle \neq \varnothing$. So $\rho_{H}\langle y\rangle=\rho_{H}\langle z\rangle$, because $H$ is a strong subset. Now let $t_{2}$ be an arbitrary translation of $\rho_{X}\langle y\rangle$. Then $t_{2}(y) \in X$, i.e., $t_{2}(y) \in \rho_{H}^{\circ}\langle t\rangle$. Therefore, $t\left(t_{2}(y)\right) \in H$, which gives $t \circ t_{2} \in \rho_{H}\langle y\rangle=\rho_{H}\langle z\rangle$. So, $t \circ t_{2} \in \rho_{H}\langle z\rangle$, i.e., $t\left(t_{2}(z)\right) \in H$. This implies $t_{2}(z) \in \rho_{H}^{\circ}\langle t\rangle$, which means that $t_{2}(z) \in X$. Thus, $t_{2} \in \rho_{X}\langle z\rangle$. Therefore, $\rho_{X}\langle y\rangle \subseteq \rho_{X}\langle z\rangle$. Similarly, we show that $\rho_{X}\langle z\rangle \subseteq \rho_{X}\langle y\rangle$. So, $\rho_{X}\langle y\rangle=\rho_{X}\langle z\rangle$. Hence, $\rho_{X}\langle y\rangle \cap \rho_{X}\langle z\rangle \neq \varnothing$ implies $\rho_{X}\langle y\rangle=\rho_{X}\langle z\rangle$. This means that $X$ is a strong subset.

Now assume that $(a, b) \in \mathcal{R}_{H}$, i.e., $\rho_{H}\langle a\rangle=\rho_{H}\langle b\rangle$. Let $t_{3} \in \rho_{X}\langle a\rangle$, where $t_{3} \in T_{n}(G)$. Then $t_{3}(a) \in X$, i.e., $t_{3}(a) \in \rho_{H}^{\circ}\langle t\rangle$. Therefore, $\left(t \circ t_{3}\right)(a) \in H$, so $t \circ t_{3} \in \rho_{H}\langle a\rangle=\rho_{H}\langle b\rangle$. Thus, $t \circ t_{3} \in \rho_{H}\langle b\rangle$, i.e., $t\left(t_{3}(b)\right) \in H$, whence $t_{3}(b) \in \rho_{H}^{\circ}\langle t\rangle$. This gives $t_{3}(b) \in X$. Consequently, $t_{3} \in \rho_{X}\langle b\rangle$. Therefore, $\rho_{X}\langle a\rangle \subseteq \rho_{X}\langle b\rangle$. Similarly, we show that $\rho_{X}\langle b\rangle \subseteq \rho_{X}\langle a\rangle$. Thus, $\rho_{X}\langle a\rangle=\rho_{X}\langle b\rangle$, i.e., $(a, b) \in \mathcal{R}_{X}$. So, $\mathcal{R}_{H} \subseteq \mathcal{R}_{X}$.

From this inclusion it follows that $W_{H}$ is contained in some $\mathcal{R}_{X}$-class. Let $a \in W_{H}$, i.e., $\rho_{H}\langle a\rangle=\varnothing$. Then also $\rho_{X}\langle a\rangle=\varnothing$. Indeed, $\rho_{X}\langle a\rangle \neq \varnothing$ means that $t_{4}(a) \in X=\rho_{H}^{\circ}\langle t\rangle$ for some $t_{4} \in \rho_{X}\langle a\rangle$. So, $t_{4}(a) \in \rho_{H}^{\circ}\langle t\rangle$, which implies $t\left(t_{4}(a)\right) \in H$. Thus, $\left(t \circ t_{4}\right)(a) \in H$, and consequently, $t \circ t_{4} \in \rho_{H}\langle a\rangle$. Hence, $\rho_{H}\langle a\rangle \neq \varnothing$, i.e., $a \notin W_{H}$. This impossible because by the assumption $a \in W_{H}$. Obtained contradiction proves that $\rho_{X}\langle a\rangle=\varnothing$. Consequently, $a \in W_{X}$ and $W_{H} \subseteq W_{X}$.

Since $\mathcal{R}_{H} \subseteq \mathcal{R}_{X}$, the set $W_{X}$ is a union of $\mathcal{R}_{H}$-classes. To prove that $\mathcal{R}_{H}$ and $\mathcal{R}_{X}^{*}$ are coincide on the set $G \backslash W_{X}$ it suffices to show that $(a, b) \in \mathcal{R}_{X}$ implies $(a, b) \in \mathcal{R}_{H}$ for all $a, b \in G \backslash W_{X}$. Let $(a, b) \in \mathcal{R}_{X}$ for some $a, b \in G \backslash W_{X}$. Then $\rho_{X}\langle a\rangle=\rho_{X}\langle b\rangle \neq \varnothing$, so there exists a translation $t_{5} \in T_{n}(G)$ such that 
$t_{5}(a)$ and $t_{5}(b)$ are in $X=\rho_{H}^{\circ}\langle t\rangle$. Therefore, $t\left(t_{5}(a)\right)$ and $t\left(t_{5}(b)\right)$ are in $H$. So, $\left(t \circ t_{5}\right)(a)$ and $\left(t \circ t_{5}\right)(b)$ also are in $H$. Thus, $t \circ t_{5} \in \rho_{H}\langle a\rangle \cap \rho_{H}\langle b\rangle$, which shows $\rho_{H}\langle a\rangle \cap \rho_{H}\langle b\rangle \neq \varnothing$. Since $H$ is strong, the last implies $\rho_{H}\langle a\rangle=\rho_{H}\langle b\rangle$. Therefore, $(a, b) \in \mathcal{R}_{H}$. Thus, $\mathcal{R}_{X}^{*}=\mathcal{R}_{H} \cap\left(G \backslash W_{X}\right) \times\left(G \backslash W_{X}\right)$.

Corollary 2.12. Let $H$ be a strong subset of a Menger algebra $(G, o)$. Then, for any translation $t \in T_{n}(G)$, the set $\rho_{H}^{\circ}\langle t\rangle$ is strong.

Proof. It was noted above that the empty subset is strong, so if for some $t \in$ $T_{n}(G)$, we have $\rho_{H}^{\circ}\langle t\rangle=\varnothing$, then, obviously, $\rho_{H}^{\circ}\langle t\rangle$ is strong. If $\rho_{H}^{\circ}\langle t\rangle \neq \varnothing$, then $\rho_{H}^{\circ}\langle t\rangle$ is an $\mathcal{R}_{H}$-class different from $W_{H}$ (Proposition 2.10). Therefore, by Theorem 2.11, $\rho_{H}^{\circ}\langle t\rangle$ is a strong subset of $(G, o)$.

Proposition 2.13. If a strong subset $H$ of a Memger algebra $(G, o)$ is its l-ideal, then $\mathcal{R}_{H}=\mathcal{R}_{X}$ for any $X \neq W_{H}$.

Proof. Let $H$ be a strong subset of $(G, o)$. By Theorem 2.11, we have $W_{H} \subseteq W_{X}$, $\mathcal{R}_{H} \subseteq \mathcal{R}_{X}$ and $\mathcal{R}_{X}^{*}=\mathcal{R}_{H} \cap\left(G \backslash W_{X}\right) \times\left(G \backslash W_{X}\right)$. Thus, to prove $\mathcal{R}_{H}=\mathcal{R}_{X}$ it remains to show that $W_{H}=W_{X}$. Due to the above it is sufficient to show only $W_{X} \subseteq W_{H}$.

Let $a \in W_{X}$. Then $\rho_{X}\langle a\rangle=\varnothing$, i.e., $t(a) \notin X$ for all $t \in T_{n}(G)$. Since $X \neq$ $W_{H}$, for some $t_{1} \in T_{n}(G)$ we have $X=\rho_{H}^{\circ}\left\langle t_{1}\right\rangle$ (Proposition 2.10). Therefore, $t(a) \notin \rho_{H}^{\circ}\left\langle t_{1}\right\rangle$ for all $t \in T_{n}(G)$. Thus,

$$
\left(t_{1} \circ t\right)(a) \notin H \text { for all } t \in T_{n}(G) .
$$

Suppose that $a \notin W_{H}$, i.e., $\rho_{H}\langle a\rangle \neq \varnothing$. Then $t_{2}(a) \in H$ for some $t_{2} \in T_{n}(G)$, whence $t_{1}\left(t_{2}(a)\right) \in t_{1}(H)$. So, $\left(t_{1} \circ t_{2}\right)(a) \in t_{1}(H) \subseteq H$ since $H$ is an $l$-ideal. Hence $\left(t_{1} \circ t_{2}\right)(a) \in H$, which contradics to (10). Therefore $a \in W_{H}$ and $W_{X} \subseteq W_{H}$. This completes the proof.

We say that a $v$-congruence $\varepsilon$ on a Menger algebra $(G, o)$ is partially $v$ cancellative with v-residue $W$, if $W$ is an $\varepsilon$-class of $(G, o)$ and

$$
\left(u\left[\left.\bar{w}\right|_{i} g_{1}\right], u\left[\left.\bar{w}\right|_{i} g_{2}\right]\right) \in \varepsilon \wedge u\left[\left.\bar{w}\right|_{i} g_{1}\right] \notin W \longrightarrow\left(g_{1}, g_{2}\right) \in \varepsilon
$$

for all $u, g_{1}, g_{2} \in G, \bar{w} \in G^{n}, i=1, \ldots, n$, i.e., if $\varepsilon \cap(G \backslash W) \times(G \backslash W)$ is $v$-cancellative. In the case when $W$ is the empty set we obtain a $v$-cancellative relation.

Proposition 2.14. A principal v-congruence $\mathcal{R}_{H}$ on a Menger algebra $(G, o)$ is partially $v$-cancellative with $v$-residue $W_{H}$ if and only if for any translation $t \in T_{n}(G)$ the following implication

$$
\left(t\left(g_{1}\right), t\left(g_{2}\right)\right) \in \mathcal{R}_{H} \wedge t\left(g_{1}\right) \notin W_{H} \longrightarrow\left(g_{1}, g_{2}\right) \in \mathcal{R}_{H}
$$

is true. 
Proof. If $\mathcal{R}_{H}$ and $W_{H}$ satisfy (11), then for $t(x)=u\left[\left.\bar{w}\right|_{i} x\right]$, where $u \in G$, $\bar{w} \in G^{n}, i \in\{1, \ldots, n\}$, we obtain

$$
\left(u\left[\left.\bar{w}\right|_{i} g_{1}\right], u\left[\left.\bar{w}\right|_{i} g_{2}\right]\right) \in \mathcal{R}_{H} \wedge u\left[\left.\bar{w}\right|_{i} g_{1}\right] \notin W_{H} \longrightarrow\left(g_{1}, g_{2}\right) \in \mathcal{R}_{H},
$$

which shows that $\mathcal{R}_{H}$ is partially $v$-cancellative with $v$-residue $W_{H}$.

Conversely, let (12) and the premise of (11) be satisfied for $t \in T_{n}(G)$ of the form $t(x)=u\left[\left.\bar{w}\right|_{i} t_{1}(x)\right]$, where $t_{1} \in T_{n}(G), u \in G, \bar{w} \in G^{n}, i \in\{1, \ldots, n\}$. Then, $\left(u\left[\left.\bar{w}\right|_{i} t_{1}\left(g_{1}\right)\right], u\left[\left.\bar{w}\right|_{i} t_{1}\left(g_{2}\right)\right]\right) \in \mathcal{R}_{H}$ and $u\left[\left.\bar{w}\right|_{i} t_{1}\left(g_{1}\right)\right] \notin W_{H}$, hence by (12), we get $\left(t_{1}\left(g_{1}\right), t_{1}\left(g_{2}\right)\right) \in \mathcal{R}_{H}$. Since $W_{H}$ is an $l$-ideal, from $u\left[\left.\bar{w}\right|_{i} t_{1}\left(g_{1}\right)\right] \notin W_{H}$ we obtain $t_{1}\left(g_{1}\right) \notin W_{H}$. So, $\left(t_{1}\left(g_{1}\right), t_{1}\left(g_{2}\right)\right) \in \mathcal{R}_{H}$ and $t_{1}\left(g_{1}\right) \notin W_{H}$. Continuing a similar argumentation after a finite number of steps we obtain $\left(g_{1}, g_{2}\right) \in \mathcal{R}_{H}$. Thus, the condition (11) is proved.

Theorem 2.15. A nonempty subset $H$ of a Menger algebra $(G, o)$ is strong if and only if it has the following two properties:

(i) $\rho_{H}\left\langle h_{1}\right\rangle \cap \rho_{H}\left\langle h_{2}\right\rangle \neq \varnothing$ for all $h_{1}, h_{2} \in H$,

(ii) $\mathcal{R}_{H}$ is partially $v$-cancellative with v-residue $W_{H}$.

Proof. If a nonempty subset $H$ of $(G, o)$ is strong, then, by Proposition 2.9. it is an $\mathcal{R}_{H}$-class different from $W_{H}$. Since $H \neq W_{H}$, by Proposition 2.10, there exists a translation $t \in T_{n}(G)$ such that $H=\rho_{H}^{\circ}\langle t\rangle$. So, $t\left(h_{1}\right) \in H$ and $t\left(h_{2}\right) \in H$ for any $h_{1}, h_{2} \in H$. Thus, $t \in \rho_{H}\left\langle h_{1}\right\rangle \cap \rho_{H}\left\langle h_{2}\right\rangle$, which implies $\rho_{H}\left\langle h_{1}\right\rangle \cap \rho_{H}\left\langle h_{2}\right\rangle \neq \varnothing$. This proves the first property.

Now suppose that $\left(u\left[\left.\bar{w}\right|_{i} g_{1}\right], u\left[\left.\bar{w}\right|_{i} g_{2}\right]\right) \in \mathcal{R}_{H}$ and $u\left[\left.\bar{w}\right|_{i} g_{1}\right] \notin W_{H}$ for some $u, g_{1}, g_{2} \in G, \bar{w} \in G^{n}, i=1, \ldots, n$. Since $u\left[\left.\bar{w}\right|_{i} g_{1}\right] \notin W_{H}$ means that $t_{1}\left(u\left[\bar{w} \mid{ }_{i} g_{1}\right]\right) \in H$ for some $t_{1} \in T_{n}(G)$, we have $t_{1}\left(u\left[\bar{w} \mid{ }_{i} g_{2}\right]\right) \in H$. If $t\left(g_{1}\right) \in H$ for some $t \in T_{n}(G)$, then from $t_{1}\left(u\left[\left.\bar{w}\right|_{i} g_{2}\right]\right), t_{1}\left(u\left[\left.\bar{w}\right|_{i} g_{1}\right]\right), t\left(g_{1}\right) \in H$ we obtain $t\left(g_{2}\right) \in H$ because $H$ is a strong subset. So, $t\left(g_{1}\right) \in H$ implies $t\left(g_{2}\right) \in H$. Analogously $t\left(g_{2}\right) \in H$ implies $t\left(g_{1}\right) \in H$. Thus $\left(g_{1}, g_{2}\right) \in \mathcal{R}_{H}$, which proves the second property.

Conversely, let $H$ be an arbitrary nonempty subset of $(G, o)$ satisfying $(i)$ and (ii). If $\rho_{H}\langle a\rangle \cap \rho_{H}\langle b\rangle \neq \varnothing$ for some $a, b \in G$, then for some $t \in T_{n}(G)$ we have $t \in \rho_{H}\langle a\rangle$ and $t \in \rho_{H}\langle b\rangle$. Thus $t(a) \in H$ and $t(b) \in H$. But $H \cap W_{H}=\varnothing$, so $t(a) \notin W_{H}$ and $t(b) \notin W_{H}$. This, by $(i)$, gives $\rho_{H}\langle t(a)\rangle \cap \rho_{H}\langle t(b)\rangle \neq \varnothing$. Hence, there is a translation $t_{1} \in \rho_{H}\langle t(a)\rangle \cap \rho_{H}\langle t(b)\rangle$. For this translation $t_{1}(t(a)) \in H$ and $t_{1}(t(b)) \in H$. Consequently, $t(a) \in \rho_{H}^{\circ}\left\langle t_{1}\right\rangle$ and $t(b) \in \rho_{H}^{\circ}\left\langle t_{1}\right\rangle$, whence $(t(a), t(b)) \in \mathcal{R}_{H}$ because, by Proposition 2.10 $\rho_{H}^{\circ}\left\langle t_{1}\right\rangle$ is an $\mathcal{R}_{H}$-class different from $W_{H}$. This, by $(i i)$, implies $(a, b) \in \mathcal{R}_{H}$. Therefore, $\rho_{H}\langle a\rangle=\rho_{H}\langle b\rangle$, which shows that a subset $H$ is strong.

We say that a subset $X$, possibly empty, of a Menger algebra $(G, o)$ is $l$ consistent in $(G, o)$, if for any $g \in G$ and $t \in T_{n}(G) t(g) \in X$ implies $g \in X$.

Proposition 2.16. Let $H$ be a strong subset of a Menger algebra $(G, o)$. Then $\mathcal{R}_{H}$ is a v-cancellative $v$-congruence if and only if $W_{H}$ is l-consistent in $(G, o)$. 
Proof. Let $H$ be a strong subset of $(G, o)$. Then, according to the second condition of Theorem 2.15, the relation $\mathcal{R}_{H}$ is partially $v$-cancellative with $v$-residue $W_{H}$, i.e., the implication (11) is satisfied. Suppose that $t(a), t(b) \in W_{H}$. If $W_{H}$ is $l$-consistent in $(G, o)$, then $a, b \in W_{H}$, so $(a, b) \in \mathcal{R}_{H}$. Thus, $\mathcal{R}_{H}$ satisfies (4), so it is $v$-cancellative.

Conversely, let $\mathcal{R}_{H}$ be a $v$-cancellative $v$-congruence. If $t(a) \in W_{H}$ for some $t \in T_{n}(G)$, then $t_{1}(t(a)) \notin H$ for all $t_{1} \in T_{n}(G)$. So, for all $t_{1} \in T_{n}(G)$ we have $\left(t_{1} \circ t\right)(t(a)) \notin H$. Thus $t_{1}(t(t(a))) \notin H$, which implies $t(t(a)) \in W_{H}$. Hence, $(t(a), t(t(a))) \in \mathcal{R}_{H}$, consequently $(a, t(a)) \in \mathcal{R}_{H}$. Therefore $a \in W_{H}$. This shows that $W_{H}$ is $l$-consistent in $(G, o)$.

\section{Principal l-congruences on Menger algebras}

For a nonempty subset $H$ of a Menger algebra $(G, o)$ of rank $n$ and $B=G^{n} \cup\{\bar{e}\}$ we define the following subsets:

$$
\begin{gathered}
\eta_{H}=\{(g, \bar{x}) \in G \times B \mid g[\bar{x}] \in H\}, \\
\eta_{H}\langle g\rangle=\{\bar{x} \in B \mid g[\bar{x}] \in H\}, \\
\eta_{H}^{\circ}\langle\bar{x}\rangle=\{g \in G \mid g[\bar{x}] \in H\}, \\
H W=\left\{g \in G \mid \eta_{H}\langle g\rangle=\varnothing\right\}, \\
\mathcal{L}_{H}=\left\{\left(g_{1}, g_{2}\right) \in G \times G \mid \eta_{H}\left\langle g_{1}\right\rangle=\eta_{H}\left\langle g_{2}\right\rangle\right\} .
\end{gathered}
$$

It is easy to see that $\mathcal{L}_{H}$ is an equivalence relation on $(G, o)$ and ${ }_{H} W$ is its an equivalence class, provided that it is not empty. It is also easy show that

$$
\begin{gathered}
\left(g_{1}, g_{2}\right) \in \mathcal{L}_{H} \longleftrightarrow(\forall \bar{x} \in B)\left(g_{1}[\bar{x}] \in H \longleftrightarrow g_{2}[\bar{x}] \in H\right), \\
g \in_{H} W \longleftrightarrow(\forall \bar{x} \in B) g[\bar{x}] \notin H .
\end{gathered}
$$

${ }_{H} W$ is called an $l$-residue of $H$. Obviously ${ }_{H} W \cap H=\varnothing$.

Proposition 3.1. For any subset $H$ of a Menger algebra $(G, o)$ the relation $\mathcal{L}_{H}$ is an l-congruence. Moreover, ${ }_{H} W$ is an $s$-ideal of $(G, o)$, provided that ${ }_{H} W \neq \varnothing$.

Proof. Let $\left(g_{1}, g_{2}\right) \in \mathcal{L}_{H}$, then for all $\bar{x}, \bar{y} \in B$ we have

$$
g_{1}[\bar{y} * \bar{x}] \in H \longleftrightarrow g_{2}[\bar{y} * \bar{x}] \in H,
$$

whence we obtain

$$
\left(g_{1}[\bar{y}]\right)[\bar{x}] \in H \longleftrightarrow\left(g_{2}[\bar{y}]\right)[\bar{x}] \in H
$$

for all $\bar{x}, \bar{y} \in B$. This means that $\left(g_{1}[\bar{y}], g_{2}[\bar{y}]\right) \in \mathcal{L}_{H}$ for any $\bar{y} \in B$. Thus, $\mathcal{L}_{H}$ is an $l$-regular, consequently, it is an $l$-congruence.

Let $g \in_{H} W$, then $g[\bar{y} * \bar{x}] \notin H$ for all $\bar{y} * \bar{x}$, where $\bar{x}, \bar{y} \in B$. Therefore, $(\forall \bar{x} \in B)(g[\bar{y}])[\bar{x}] \notin H$ for every $\bar{y} \in B$. Thus, $g[\bar{y}] \in_{H} W$ for every $\bar{y} \in B$. So, ${ }_{H} W$ is an $s$-ideal of $(G, o)$. 

$H$.

Further the relation $\mathcal{L}_{H}$ will be called a principal l-congruence induced by

Proposition 3.2. Any l-congruence $\varepsilon$ on a Menger algebra $(G, o)$ has the form

$$
\varepsilon=\bigcap\left\{\mathcal{L}_{H} \mid H \in G / \varepsilon\right\},
$$

where $G / \varepsilon$ is the set of all $\varepsilon$-classes.

Proof. Let $\left(g_{1}, g_{2}\right) \in \varepsilon$ and $g_{1}[\bar{x}] \in H$ for some $\bar{x} \in B$ and $H \in G / \varepsilon$. Since $\varepsilon$ is an $l$-regular relation, for any $\bar{x} \in B$ we have $\left(g_{1}[\bar{x}], g_{2}[\bar{x}]\right) \in \varepsilon$. But $H$ is an $\varepsilon$-class and $g_{1}[\bar{x}] \in H$, then, obviously, $g_{2}[\bar{x}] \in H$. Analogously, $g_{2}[\bar{x}] \in H$ implies $g_{1}[\bar{x}] \in H$. Thus,

$$
(\forall \bar{x} \in B)\left(g_{1}[\bar{x}] \in H \longleftrightarrow g_{2}[\bar{x}] \in H\right),
$$

i.e., $\left(g_{1}, g_{2}\right) \in \mathcal{L}_{H}$. Since $H$ is an arbitrary $\varepsilon$-class, from the abowe we obtain inclusion $\varepsilon \subseteq \bigcap\left\{\mathcal{L}_{H} \mid H \in G / \varepsilon\right\}$.

To prove the converse inclusion consider an arbitrary pair $\left(g_{1}, g_{2}\right) \in$ $\bigcap\left\{\mathcal{L}_{H} \mid H \in G / \varepsilon\right\}$. Then, $g_{1}[\bar{x}] \in H$ if and only if $g_{2}[\bar{x}] \in H$ for any $\bar{x} \in B$ and $H \in G / \varepsilon$. This, for $\bar{x}=\bar{e}$ and $H=\left\langle g_{1}\right\rangle$, where $\left\langle g_{1}\right\rangle$ denotes the $\varepsilon$ class determined by $g_{1}$, gives $g_{2} \in\left\langle g_{1}\right\rangle$ Thus, $\left(g_{1}, g_{2}\right) \in \varepsilon$ and consequently $\bigcap\left\{\mathcal{L}_{H} \mid H \in G / \varepsilon\right\} \subseteq \varepsilon$, which completes the proof.

Definition 3.3. A subset $H$ of a Menger algebra $(G, o)$ is called $l$-strong if

$$
\eta_{H}\langle a\rangle \cap \eta_{H}\langle b\rangle \neq \varnothing \longrightarrow \eta_{H}\langle a\rangle=\eta_{H}\langle b\rangle
$$

for all $a, b \in G$.

Theorem 3.4. For a subset $H$ of a Menger algebra $(G, o)$ the following conditions are equivalent.

(i) $H$ is l-strong.

(ii) For all $g_{1}, g_{2} \in G$ and $\bar{x}, \bar{y} \in B$

$$
g_{1}[\bar{x}] \in H \wedge g_{2}[\bar{x}] \in H \wedge g_{2}[\bar{y}] \in H \longrightarrow g_{1}[\bar{y}] \in H .
$$

(iii) For all $\bar{x}, \bar{y} \in B$

$$
\eta_{H}^{\circ}\langle\bar{x}\rangle \cap \eta_{H}^{\circ}\langle\bar{y}\rangle \neq \varnothing \longrightarrow \eta_{H}^{\circ}\langle\bar{x}\rangle=\eta_{H}^{\circ}\langle\bar{y}\rangle .
$$

Proof. $(i) \longrightarrow($ ii $)$ Let $H$ be a $l$-strong subset of $(G, o)$ and $g_{1}[\bar{x}], g_{2}[\bar{x}], g_{2}[\bar{y}] \in H$ for some $g_{1}, g_{2} \in G, \bar{x}, \bar{y} \in B$. Then $g_{1}[\bar{x}], g_{2}[\bar{x}] \in H$ imply $\bar{x} \in \eta_{H}\left\langle g_{1}\right\rangle \cap \eta_{H}\left\langle g_{2}\right\rangle$, so $\eta_{H}\left\langle g_{1}\right\rangle \cap \eta_{H}\left\langle g_{2}\right\rangle \neq \varnothing$. This, according to (13), gives $\eta_{H}\left\langle g_{1}\right\rangle=\eta_{H}\left\langle g_{2}\right\rangle$. From $g_{2}[\bar{y}] \in H$ we obtain $\bar{y} \in \eta_{H}\left\langle g_{2}\right\rangle$, which means that $\bar{y} \in \eta_{H}\left\langle g_{1}\right\rangle$. Thus, $g_{1}[\bar{y}] \in H$. This proves $(i i)$. 
(ii) $\longrightarrow$ (iii) If $\eta_{H}^{\circ}\langle\bar{x}\rangle \cap \eta_{H}^{\circ}\langle\bar{y}\rangle \neq \varnothing$, then for any $g \in \eta_{H}^{\circ}\langle\bar{x}\rangle \cap \eta_{H}^{\circ}\langle\bar{y}\rangle$ we have $g[\bar{x}] \in H$ and $g[\bar{y}] \in H$. If $g_{1} \in \eta_{H}^{\circ}\langle\bar{x}\rangle$, then $g_{1}[\bar{x}] \in H$. Thus, $g_{1}[\bar{x}], g[\bar{x}], g[\bar{y}] \in$ $H$, whence, by (14), we obtain $g_{1}[\bar{y}] \in H$. Thus $g_{1} \in \eta_{H}^{\circ}\langle\bar{y}\rangle$. So, $\eta_{H}^{\circ}\langle\bar{x}\rangle \subseteq \eta_{H}^{\circ}\langle\bar{y}\rangle$. Analogously, $\eta_{H}^{\circ}\langle\bar{y}\rangle \subseteq \eta_{H}^{\circ}\langle\bar{x}\rangle$. Hence, $\eta_{H}^{\circ}\langle\bar{x}\rangle=\eta_{H}^{\circ}\langle\bar{y}\rangle$. This proves $(i i i)$.

$($ iii $) \longrightarrow(i)$ If $\eta_{H}\langle a\rangle \cap \eta_{H}\langle b\rangle \neq \varnothing$, then for some $\bar{x} \in B$ we have $a[\bar{x}] \in H$ and $b[\bar{x}] \in H$. If $\bar{y} \in \eta_{H}\langle a\rangle$, then $a[\bar{y}] \in H$, so from $a[\bar{x}] \in H$ and $a[\bar{y}] \in H$ we obtain $a \in \eta_{H}^{\circ}\langle\bar{x}\rangle \cap \eta_{H}^{\circ}\langle\bar{y}\rangle$. Consequently, $\eta_{H}^{\circ}\langle\bar{x}\rangle \cap \eta_{H}^{\circ}\langle\bar{y}\rangle \neq \varnothing$, whence, by (15), we deduce $\eta_{H}^{\circ}\langle\bar{x}\rangle=\eta_{H}^{\circ}\langle\bar{y}\rangle$. Since $b[\bar{x}] \in H$, we have $b \in \eta_{H}^{\circ}\langle\bar{x}\rangle$, so $b \in \eta_{H}^{\circ}\langle\bar{y}\rangle$, i.e., $b[\bar{y}] \in H$. Thus $\bar{y} \in \eta_{H}\langle b\rangle$. Therefore $\eta_{H}\langle a\rangle \subseteq \eta_{H}\langle b\rangle$. Similarly we can show that $\eta_{H}\langle b\rangle \subseteq \eta_{H}\langle a\rangle$. Hence $\eta_{H}\langle a\rangle=\eta_{H}\langle b\rangle$, which means that $H$ is $l$-strong.

Note that according to (14) the empty subset is $l$-strong.

Proposition 3.5. Each nonempty normal l-complex $H$ of a Menger algebra $(G, o)$ is an $\mathcal{L}_{H}$-equivalence class different from l-residue ${ }_{H} W$.

Proof. Let $h_{1}, h_{2} \in H$ and $h_{1}[\bar{x}] \in H$ for some $\bar{x} \in B$. Then, by (2), we obtain $h_{2}[\bar{x}] \in H$. Similarly, $h_{2}[\bar{x}] \in H$ implies $h_{1}[\bar{x}] \in H$. So, for all $\bar{x} \in B$ we have $h_{1}[\bar{x}] \in H \longleftrightarrow h_{2}[\bar{x}] \in H$. Thus $\left(h_{1}, h_{2}\right) \in \mathcal{L}_{H}$. Consequently, $H$ is contained in some $\mathcal{L}_{H}$-class. Denote this class by $X$. Hence, $H \subseteq X$.

Let $g$ be an arbitrary element of $X$ and $h \in H$. Then obviously $(h, g) \in \mathcal{L}_{H}$, i.e., $h[\bar{x}] \in H \longleftrightarrow g[\bar{x}] \in H$ for all $\bar{x} \in B$. This for $\bar{x}=\bar{e}$ means that $h \in H \longleftrightarrow g \in H$, so $g \in H$. Therefore, $X \subseteq H$. Consequently, $H=X$. Since $H \cap{ }_{H} W=\varnothing$, then $X \neq{ }_{H} W$.

Corollary 3.6. Each nonempty l-strong subset $H$ of a Menger algebra $(G, o)$ is an $\mathcal{L}_{H}$-equivalence class different from l-residue ${ }_{H} W$.

Proof. Indeed, putting in (14) $\bar{x}=\bar{e}$ we can see that any $l$-strong subset is a normal $l$-complex.

Proposition 3.7. Let $H$ be an l-strong subset of a Menger algebra $(G, o)$. Then all $\mathcal{L}_{H}$-classes are nonempty members of the family

$$
\mathcal{E}=\left\{\eta_{H}^{\circ}\langle\bar{x}\rangle \mid \bar{x} \in B\right\} \cup\left\{{ }_{H} W\right\} .
$$

Proof. First, we show that $\mathcal{E}$ is a partition of $G$. Clearly, $\bigcup \mathcal{E} \subseteq G$ since ${ }_{H} W \subset G$ and $\eta_{H}^{\circ}\langle\bar{x}\rangle \subset G$ for each $\bar{x} \in B$. On the other hand, for an arbitrary element $g \in G$ we have either $(\forall \bar{x} \in B) g[\bar{x}] \notin H$, or $g[\bar{x}] \in H$ for some $\bar{x} \in B$, that is, either $g \in_{H} W$, or $g \in \eta_{H}^{\circ}\langle\bar{x}\rangle$ for some $\bar{x} \in B$. Therefore, $g \in \bigcup \mathcal{E}$, so $G \subseteq \bigcup \mathcal{E}$. Thus, $G=\bigcup \mathcal{E}$, i.e., $\mathcal{E}$ covers $G$.

Moreover, members of $\mathcal{E}$ are pairwise disjoint. Indeed, if $\eta_{H}^{\circ}\langle\bar{y}\rangle \cap_{H} W \neq \varnothing$ for some $\bar{y} \in B$, then there exists an element $g \in G$ such that $g \in \eta_{H}^{\circ}\langle\bar{y}\rangle$ and $g \in_{H} W$, i.e., $g[\bar{y}] \in H$ and $(\forall \bar{x} \in B) g[\bar{x}] \notin H$, so for $\bar{x}=\bar{y}$ we have $g[\bar{y}] \in H$ and $g[\bar{y}] \notin H$, which is impossible. Thus, $\eta_{H}^{\circ}\langle\bar{y}\rangle \cap_{H} W=\varnothing$ for every $\bar{y} \in B$. In the case $\eta_{H}^{\circ}\langle\bar{x}\rangle \cap \eta_{H}^{\circ}\langle\bar{y}\rangle \neq \varnothing$ we obtain $\eta_{H}^{\circ}\langle\bar{x}\rangle=\eta_{H}^{\circ}\langle\bar{y}\rangle$ because $H$ is an $l$-strong subset. Thus, if $\eta_{H}^{\circ}\langle\bar{x}\rangle \neq \eta_{H}^{\circ}\langle\bar{y}\rangle$, then $\eta_{H}^{\circ}\langle\bar{x}\rangle \cap \eta_{H}^{\circ}\langle\bar{y}\rangle=\varnothing$, for all $\bar{x}, \bar{y} \in B$. Hence all members of $\mathcal{E}$ are pairwise disjoint subsets. Therefore $\mathcal{E}$ is a partition of $G$. 
Now let $\left(g_{1}, g_{2}\right) \in \mathcal{L}_{H}$, i.e., $\eta_{H}\left\langle g_{1}\right\rangle=\eta_{H}\left\langle g_{2}\right\rangle$. If $\eta_{H}\left\langle g_{1}\right\rangle=\varnothing$, then $g_{1}, g_{2} \in$ ${ }_{H} W$, so $g_{1}$ and $g_{2}$ are in an $\mathcal{L}_{H}$-class ${ }_{H} W$. If $\eta_{H}\left\langle g_{1}\right\rangle \neq \varnothing$, then there exists $\bar{x} \in B$ such that $\bar{x} \in \eta_{H}\left\langle g_{1}\right\rangle=\eta_{H}\left\langle g_{2}\right\rangle$, so $g_{1}[\bar{x}] \in H$ and $g_{2}[\bar{x}] \in H$, hence $g_{1}, g_{2} \in \eta_{H}^{\circ}\langle\bar{x}\rangle$.

Conversely, if $g_{1}, g_{2} \in \eta_{H}^{\circ}\langle\bar{x}\rangle$ for some $\bar{x} \in B$, then $g_{1}[\bar{x}] \in H$ and $g_{2}[\bar{x}] \in H$, so $\bar{x} \in \eta_{H}\left\langle g_{1}\right\rangle \cap \eta_{H}\left\langle g_{2}\right\rangle$. Consequently, $\eta_{H}\left\langle g_{1}\right\rangle \cap \eta_{H}\left\langle g_{2}\right\rangle \neq \varnothing$, whence $\eta_{H}\left\langle g_{1}\right\rangle=$ $\eta_{H}\left\langle g_{2}\right\rangle$, i.e., $\left(g_{1}, g_{2}\right) \in \mathcal{L}_{H}$. Thus, $\eta_{H}^{\circ}\langle\bar{x}\rangle$ is an $\mathcal{L}_{H}$-class.

Theorem 3.8. Let $H$ be a nonempty l-strong subset of a Menger algebra $(G, o)$. Then each $\mathcal{L}_{H}$-class $X \neq{ }_{H} W$ is l-strong, ${ }_{H} W \subseteq_{X} W, \mathcal{L}_{H} \subseteq \mathcal{L}_{X}$ and restrictions of $\mathcal{L}_{H}$ and $\mathcal{L}_{X}$ on $G \backslash_{X} W$ coincide.

Proof. Let $\eta_{X}\langle a\rangle \cap \eta_{X}\langle b\rangle \neq \varnothing$, then there exists $\bar{x} \in B$ such that $\bar{x} \in \eta_{X}\langle a\rangle \cap$ $\eta_{X}\langle b\rangle$. So, $a[\bar{x}] \in X$ and $b[\bar{x}] \in X$. Since $X \neq{ }_{H} W$ is an $\mathcal{L}_{H^{-}}$-class, by Proposition 3.7. we have $X=\eta_{H}^{\circ}\langle\bar{y}\rangle$ for some $\bar{y} \in B$. Consequently, $a[\bar{x}] \in \eta_{H}^{\circ}\langle\bar{y}\rangle$ and $b[\bar{x}] \in \eta_{H}^{\circ}\langle\bar{y}\rangle$, so $a[\bar{x}][\bar{y}] \in H$ and $b[\bar{x}][\bar{y}] \in H$, i.e., $a[\bar{x} * \bar{y}] \in H$ and $b[\bar{x} * \bar{y}] \in H$. Thus $\bar{x} * \bar{y} \in \eta_{H}\langle a\rangle$ and $\bar{x} * \bar{y} \in \eta_{H}\langle b\rangle$. Therefore $\eta_{H}\langle a\rangle \cap \eta_{H}\langle b\rangle \neq \varnothing$. Since $H$ is $l$ strong, the last gives $\eta_{H}\langle a\rangle=\eta_{H}\langle b\rangle$. Now let $\bar{z} \in \eta_{X}\langle a\rangle$, i.e., $a[\bar{z}] \in X=\eta_{H}^{\circ}\langle\bar{y}\rangle$. Then $a[\bar{z} * \bar{y}] \in H$, and consequently, $\bar{z} * \bar{y} \in \eta_{H}\langle a\rangle=\eta_{H}\langle b\rangle$. Thus, $\bar{z} * \bar{y} \in \eta_{H}\langle b\rangle$, so $b[\bar{z} * \bar{y}] \in H$, whence we obtain $b[\bar{z}] \in \eta_{H}^{\circ}\langle\bar{y}\rangle=X$. This means that $\bar{z} \in \eta_{X}\langle b\rangle$, so $\eta_{X}\langle a\rangle \subseteq \eta_{X}\langle b\rangle$. Similarly, we show $\eta_{X}\langle b\rangle \subseteq \eta_{X}\langle a\rangle$. Hence, $\eta_{X}\langle a\rangle=\eta_{X}\langle b\rangle$, proves that $X$ is $l$-strong.

Let $(a, b) \in \mathcal{L}_{H}$, i.e., $\eta_{H}\langle a\rangle=\eta_{H}\langle b\rangle$. If $\bar{u} \in \eta_{X}\langle a\rangle$, then $a[\bar{u}] \in X=\eta_{H}^{\circ}\langle\bar{y}\rangle$. So, $a[\bar{u} * \bar{y}] \in H$, consequently $\bar{u} * \bar{y} \in \eta_{H}\langle a\rangle$, whence $\bar{u} * \bar{y} \in \eta_{H}\langle b\rangle$, i.e., $b[\bar{u} * \bar{y}] \in H$. Thus, $b[\bar{u}] \in \eta_{H}^{\circ}\langle\bar{y}\rangle=X$, so $\bar{u} \in \eta_{X}\langle b\rangle$. Therefore, $\eta_{X}\langle a\rangle \subseteq \eta_{X}\langle b\rangle$. Similarly we obtain the reverse inclusion. Hence $\eta_{X}\langle a\rangle=\eta_{X}\langle b\rangle$. This shows that $(a, b) \in \mathcal{L}_{X}$. Therefore $\mathcal{L}_{H} \subseteq \mathcal{L}_{X}$.

Thus, ${ }_{H} W$ is contained in some $\mathcal{L}_{X}$-class. We show that it is the class ${ }_{X} W$. Obviously $\eta_{H}\langle a\rangle=\varnothing$ for any $a \in_{H} W$. Then also $\eta_{X}\langle a\rangle=\varnothing$. Indeed, if $\eta_{X}\langle a\rangle \neq \varnothing$, then for some $\bar{w} \in B$ we have $\bar{w} \in \eta_{X}\langle a\rangle$ and $a[\bar{w}] \in \eta_{H}^{\circ}\langle\bar{y}\rangle$ because $X=\eta_{H}^{\circ}\langle\bar{y}\rangle$. Thus $a[\bar{w} * \bar{y}] \in H$. Consequently, $\bar{w} * \bar{y} \in \eta_{H}\langle a\rangle$, which implies $\eta_{H}\langle a\rangle \neq \varnothing$, i.e., $a \notin_{H} W$. Obtained contradiction proves that $\eta_{X}\langle a\rangle=\varnothing$. So, $a \in_{X} W$ and ${ }_{H} W \subseteq_{X} W$.

Since $\mathcal{L}_{H} \subseteq \mathcal{L}_{X}$, the set ${ }_{X} W$ is a union of some $\mathcal{L}_{H}$-classes. We show that restrictions of $\mathcal{L}_{H}$ and $\mathcal{L}_{X}$ on $G \backslash_{X} W$ coincide. For this we show that for all $a, b \in G \backslash_{X} W$ such that $(a, b) \in \mathcal{L}_{X}$ we have $(a, b) \in \mathcal{L}_{H}$. In fact, if $a, b \in G \backslash_{X} W$ and $(a, b) \in \mathcal{L}_{X}$, then $\eta_{H}\langle a\rangle=\eta_{H}\langle b\rangle \neq \varnothing$. Thus $a[\bar{v}] \in X$ and $b[\bar{v}] \in X$ for some $\bar{v} \in B$, that is, $a[\bar{v}] \in \eta_{H}^{\circ}\langle\bar{y}\rangle$ and $b[\bar{v}] \in \eta_{H}^{\circ}\langle\bar{y}\rangle$. Therefore, $a[\bar{v} * \bar{y}] \in H$ and $b[\bar{v} * \bar{y}] \in H$, which implies $\bar{v} * \bar{y} \in \eta_{H}\langle a\rangle \cap \eta_{H}\langle b\rangle$. So, $\eta_{H}\langle a\rangle \cap \eta_{H}\langle b\rangle \neq \varnothing$. Since $H$ is $l$-strong, the last implies $\eta_{H}\langle a\rangle=\eta_{H}\langle b\rangle$, i.e., $(a, b) \in \mathcal{L}_{H}$. Thus,

$$
\mathcal{L}_{H} \cap\left(G \backslash_{X} W\right) \times\left(G \backslash_{X} W\right)=\mathcal{L}_{X} \cap\left(G \backslash_{X} W\right) \times\left(G \backslash_{X} W\right),
$$

as required.

Corollary 3.9. If $H$ is an l-strong subset of a Menger algebra $(G, o)$, then for every $\bar{x} \in B$ the set $\eta_{H}^{\circ}\langle\bar{x}\rangle$ is l-strong. 
Proof. Since the empty subset is $l$-strong, so if $\eta_{H}^{\circ}\langle\bar{x}\rangle=\varnothing$ for some $\bar{x} \in B$, then, obviously, $\eta_{H}^{\circ}\langle\bar{x}\rangle$ is $l$-strong. If $\eta_{H}^{\circ}\langle\bar{x}\rangle \neq \varnothing$, then $\eta_{H}^{\circ}\langle\bar{x}\rangle$ is an $\mathcal{L}_{H}$-class different from ${ }_{H} W$ (Proposition 3.7). Therefore, by Theorem 3.8, $\eta_{H}^{\circ}\langle\bar{x}\rangle$ is $l$-strong.

Proposition 3.10. Each $\varepsilon$-class $X$ of an l-cancellative l-congruence on a Menger algebra $(G, o)$ is l-strong and $\varepsilon \subseteq \mathcal{L}_{X}$. Moreover, $\varepsilon$ and $\mathcal{L}_{X}$ coincide on $G \backslash{ }_{X} W$.

Proof. Let $\eta_{X}\langle a\rangle \cap \eta_{X}\langle b\rangle \neq \varnothing$, then $a[\bar{x}] \in X$ and $b[\bar{x}] \in X$ for some $\bar{x} \in B$. Thus, $(a[\bar{x}], b[\bar{x}]) \in \varepsilon$, whence by $l$-cancellativity we obtain $(a, b) \in \varepsilon$. This, in view of Proposition 3.2, gives $\varepsilon \subseteq \mathcal{L}_{X}$. Therefore, $(a, b) \in \mathcal{L}_{X}$, so $\eta_{X}\langle a\rangle=\eta_{X}\langle b\rangle$. Thus, $X$ is an $l$-strong subset of $(G, o)$.

Since $\varepsilon \subseteq \mathcal{L}_{X}$, we have

$$
\varepsilon \cap\left(G \backslash_{X} W\right) \times\left(G \backslash_{X} W\right) \subseteq \mathcal{L}_{X} \cap\left(G \backslash_{X} W\right) \times\left(G \backslash_{X} W\right) .
$$

Conversely, if $(a, b) \in \mathcal{L}_{X}$ and $a, b \in G \backslash_{X} W$, then

$$
(\forall \bar{x} \in B)(a[\bar{x}] \in X \longleftrightarrow b[\bar{x}] \in X) .
$$

Since $a \notin_{X} W$, we have $a[\bar{y}] \in X$ for some $\bar{y} \in B$, the above means that also $b[\bar{y}] \in X$. Consequently, $(a[\bar{y}], b[\bar{y}]) \in \varepsilon$. From this, by l-cancellativity, we deduce $(a, b) \in \varepsilon$. So,

$$
\mathcal{L}_{X} \cap\left(G \backslash_{X} W\right) \times\left(G \backslash_{X} W\right) \subseteq \varepsilon \cap\left(G \backslash_{X} W\right) \times\left(G \backslash_{X} W\right) .
$$

Comparing these two inclusions we obtain

$$
\varepsilon \cap\left(G \backslash_{X} W\right) \times\left(G \backslash_{X} W\right)=\mathcal{L}_{X} \cap\left(G \backslash_{X} W\right) \times\left(G \backslash_{X} W\right) .
$$

This means that $\varepsilon$ and $\mathcal{L}_{X}$ coincide on the set $G \backslash_{X} W$.

\section{Principal congruences on Menger algebras}

For a nonempty subset $H$ of a Menger algebra $(G, o)$ of rank $n$ and $B=G^{n} \cup\{\bar{e}\}$ consider the following subsets:

$$
\begin{gathered}
\sigma_{H}=\left\{(g,(\bar{x}, t)) \in G \times\left(B \times T_{n}(G)\right) \mid t(g[\bar{x}]) \in H\right\}, \\
\sigma_{H}\langle g\rangle=\left\{(\bar{x}, t) \in B \times T_{n}(G) \mid t(g[\bar{x}]) \in H\right\}, \\
\sigma_{H}^{\circ}\langle\bar{x}, t\rangle=\{g \in G \mid t(g[\bar{x}]) \in H\}, \\
W^{H}=\left\{g \in G \mid \sigma_{H}\langle g\rangle=\varnothing\right\}, \\
\mathcal{P}_{H}=\left\{\left(g_{1}, g_{2}\right) \in G \times G \mid \sigma_{H}\left\langle g_{1}\right\rangle=\sigma_{H}\left\langle g_{2}\right\rangle\right\} .
\end{gathered}
$$


It is clear that $\mathcal{P}_{H}$ is an equivalence relation on $(G, o)$ and $W^{H}$ is its an equivalence class, provided that it is not empty. Obviously, for any $g_{1}, g_{2}, g \in G$ we have:

$$
\begin{gathered}
\left(g_{1}, g_{2}\right) \in \mathcal{P}_{H} \longleftrightarrow\left(\forall t \in T_{n}(G)\right)(\forall \bar{x} \in B)\left(t\left(g_{1}[\bar{x}]\right) \in H \longleftrightarrow t\left(g_{2}[\bar{x}]\right) \in H\right), \\
g \in W^{H} \longleftrightarrow\left(\forall t \in T_{n}(G)\right)(\forall \bar{x} \in B) t(g[\bar{x}]) \notin H .
\end{gathered}
$$

The set $W^{H}$ is called biresidue of $H$. Note that $H \cap W^{H}=\varnothing$ since in the case $H \cap W^{H} \neq \varnothing$ there is an element $g \in G$ such that $g \in H$ and $t(g[\bar{x}]) \notin H$ for all $t \in T_{n}(G), \bar{x} \in B$. So, for the identity translation $t$ and $\bar{x}=\bar{e}$ we obtain $g \in H$ and $g \notin H$, which is impossible.

Proposition 4.1. For any subset $H$ of a Menger algebra $(G, o)$ the relation $\mathcal{P}_{H}$ is a congruence. Moreover, if the set $W^{H}$ is nonempty, then it is an sl-ideal of $(G, o)$.

Proof. Let $\left(g_{1}, g_{2}\right) \in \mathcal{P}_{H}$. Then for all $t \in T_{n}(G)$ and $\bar{x} \in B$ we have

$$
t\left(g_{1}[\bar{x}]\right) \in H \longleftrightarrow t\left(g_{2}[\bar{x}]\right) \in H .
$$

In particular, for $y_{1}, \ldots, y_{n} \in G$ we obtain $\left(y_{1}[\bar{x}], \ldots, y_{n}[\bar{x}]\right) \in B$ and

$$
t\left(g _ { 1 } [ y _ { 1 } [ \overline { x } ] \ldots y _ { n } [ \overline { x } ] ) \in H \longleftrightarrow t \left(g_{2}\left[y_{1}[\bar{x}] \ldots y_{n}[\bar{x}]\right) \in H\right.\right.
$$

whence applying (1) we get

$$
t\left(g_{1}[\bar{y}][\bar{x}]\right) \in H \longleftrightarrow t\left(g_{2}[\bar{y}][\bar{x}]\right) \in H
$$

where $\bar{y}=\left(y_{1}, \ldots, y_{n}\right)$. Thus, $\left(g_{1}[\bar{y}], g_{2}[\bar{y}]\right) \in \mathcal{P}_{H}$. So, the relation $\mathcal{P}_{H}$ is $l$-regular.

Since (16) holds for all $t \in T_{n}(G)$ and $\bar{x} \in B$, it also holds for all polynomials $t(x)=t_{1}\left(u\left[\left.\bar{w}_{0}\right|_{i} x\right]\right)$, where $t_{1} \in T_{n}(G), u \in G, \bar{w}_{0}=\left(w_{1}[\bar{x}], \ldots, w_{n}[\bar{x}]\right) \in G^{n}$, $w_{1}, \ldots, w_{n} \in G, i \in\{1, \ldots, n\}$. Therefore,

$$
t_{1}\left(u\left[\left.\bar{w}_{0}\right|_{i} g_{1}[\bar{x}]\right]\right) \in H \longleftrightarrow t_{1}\left(u\left[\left.\bar{w}_{0}\right|_{i} g_{2}[\bar{x}]\right]\right) \in H
$$

which in view of superassociativity can be rewritten in the form:

$$
t_{1}\left(u\left[\left.\bar{w}\right|_{i} g_{1}\right][\bar{x}]\right) \in H \longleftrightarrow t_{1}\left(u\left[\left.\bar{w}\right|_{i} g_{2}\right][\bar{x}]\right) \in H
$$

where $\bar{w}=\left(w_{1}, \ldots, w_{n}\right)$. So, $\left(u\left[\left.\bar{w}\right|_{i} g_{1}\right], u\left[\left.\bar{w}\right|_{i} g_{2}\right]\right) \in \mathcal{P}_{H}$ for all $i=1, \ldots, n$. Hence, $\mathcal{P}_{H}$ is a $v$-regular relation.

Thus, $\mathcal{P}_{H}$ is $l$-regular and $v$-regular, so it is stable, that is, $\mathcal{P}_{H}$ is a congruence. In a similar way we can verify that $W^{H}$ is an $s l$-ideal.

The relation $\mathcal{P}_{H}$ will be called the principal congruence induced by $H$.

Proposition 4.2. Any congruence $\varepsilon$ on a Menger algebra $(G, o)$ has the form

$$
\varepsilon=\bigcap\left\{\mathcal{P}_{H} \mid H \in G / \varepsilon\right\},
$$

where $G / \varepsilon$ is the set of all $\varepsilon$-classes. 
Proof. Let $\left(g_{1}, g_{2}\right) \in \varepsilon$ and $t\left(g_{1}[\bar{x}]\right) \in H$, where $H \in G / \varepsilon, t \in T_{n}(G)$ and $\bar{x} \in B$. As $\varepsilon$ is a congruence, from $\left(g_{1}, g_{2}\right) \in \varepsilon$, by $l$-regularity, we obtain $\left(g_{1}[\bar{x}], g_{2}[\bar{x}]\right) \in$ $\varepsilon$ for each $\bar{x} \in B$, which, by $v$-regularity, gives $\left(t\left(g_{1}[\bar{x}]\right), t\left(g_{2}[\bar{x}]\right)\right) \in \varepsilon$. Hence, $t\left(g_{1}[\bar{x}]\right)$ and $t\left(g_{2}[\bar{x}]\right)$ are in the same $\varepsilon$-class. So, $t\left(g_{2}[\bar{x}]\right) \in H$. Analogously, from $t\left(g_{2}[\bar{x}]\right) \in H$ we conclude $t\left(g_{1}[\bar{x}]\right) \in H$. Thus, $t\left(g_{1}[\bar{x}]\right) \in H \longleftrightarrow t\left(g_{2}[\bar{x}]\right) \in H$ for all $t \in T_{n}(G)$ and $\bar{x} \in B$. This means that $\left(g_{1}, g_{2}\right) \in \mathcal{P}_{H}$. Since $H$ is an arbitrary $\varepsilon$-class, the above proves the inclusion $\varepsilon \subseteq \bigcap\left\{\mathcal{P}_{H} \mid H \in G / \varepsilon\right\}$.

Conversely, if $\left(g_{1}, g_{2}\right) \in \bigcap\left\{\mathcal{P}_{H} \mid H \in G / \varepsilon\right\}$, then for all $H \in G / \varepsilon$, each $t \in T_{n}(G)$ and any $\bar{x} \in B$ we have

$$
t\left(g_{1}[\bar{x}]\right) \in H \longleftrightarrow t\left(g_{2}[\bar{x}]\right) \in H,
$$

whence for $\bar{x}=\bar{e}$ we get

$$
t\left(g_{1}\right) \in H \longleftrightarrow t\left(g_{2}\right) \in H .
$$

Since this holds for each $t \in T_{n}(G)$ and $H \in G / \varepsilon$, for the identity translation we obtain $g_{1} \in H \longleftrightarrow g_{2} \in H$. So, $g_{2} \in\left\langle g_{1}\right\rangle$, where $\left\langle g_{1}\right\rangle$ is the $\varepsilon$-class of $g_{1}$. Thus, $\left(g_{1}, g_{2}\right) \in \varepsilon$. Consequently, $\bigcap\left\{\mathcal{P}_{H} \mid H \in G / \varepsilon\right\} \subseteq \varepsilon$. This completes the proof.

Definition 4.3. A subset $H$ of a Menger algebra $(G, o)$ is called bistrong if

$$
\sigma_{H}\langle a\rangle \cap \sigma_{H}\langle b\rangle \neq \varnothing \longrightarrow \sigma_{H}\langle a\rangle=\sigma_{H}\langle b\rangle
$$

for any $a, b \in G$.

Theorem 4.4. For a subset $H$ of a Menger algebra $(G, o)$ the following conditions are equivalent.

(i) $H$ is bistrong.

(ii) For all $g_{1}, g_{2} \in G, \bar{x}, \bar{y} \in B$ and $t_{1}, t_{2} \in T_{n}(G)$

$$
t_{1}\left(g_{1}[\bar{x}]\right) \in H \wedge t_{1}\left(g_{2}[\bar{x}]\right) \in H \wedge t_{2}\left(g_{2}[\bar{y}]\right) \in H \longrightarrow t_{2}\left(g_{1}[\bar{y}]\right) \in H .
$$

(iii) For all $\bar{x}, \bar{y} \in B$ and $t_{1}, t_{2} \in T_{n}(G)$

$$
\sigma_{H}^{\circ}\left\langle\bar{x}, t_{1}\right\rangle \cap \sigma_{H}^{\circ}\left\langle\bar{y}, t_{2}\right\rangle \neq \varnothing \longrightarrow \sigma_{H}^{\circ}\left\langle\bar{x}, t_{1}\right\rangle=\sigma_{H}^{\circ}\left\langle\bar{y}, t_{2}\right\rangle .
$$

Proof. (i) $\longrightarrow($ ii $)$ Let $H$ be a bistrong subset of $(G, o)$. Suppose that $t_{1}\left(g_{1}[\bar{x}]\right), t_{1}\left(g_{2}[\bar{x}]\right), t_{2}\left(g_{2}[\bar{y}]\right) \in H$ for some $g_{1}, g_{2} \in G, \bar{x}, \bar{y} \in B$ and $t_{1}, t_{2} \in$ $T_{n}(G)$. Then from $t_{1}\left(g_{1}[\bar{x}]\right), t_{1}\left(g_{2}[\bar{x}]\right) \in H$ we obtain $\left(\bar{x}, t_{1}\right) \in \sigma_{H}\left\langle g_{1}\right\rangle \cap \sigma_{H}\left\langle g_{2}\right\rangle$, so $\sigma_{H}\left\langle g_{1}\right\rangle \cap \sigma_{H}\left\langle g_{2}\right\rangle \neq \varnothing$. This, by (17), gives $\sigma_{H}\left\langle g_{1}\right\rangle=\sigma_{H}\left\langle g_{2}\right\rangle$. But $t_{2}\left(g_{2}[\bar{y}]\right) \in H$, hence $\left(\bar{y}, t_{2}\right) \in \sigma_{H}\left\langle g_{2}\right\rangle$. Consequently, $\left(\bar{y}, t_{2}\right) \in \sigma_{H}\left\langle g_{1}\right\rangle$, i.e., $t_{2}\left(g_{1}[\bar{y}]\right) \in H$. This proves (18) and (ii).

$($ ii $) \longrightarrow($ iii $)$ Let $\sigma_{H}^{\circ}\left\langle\bar{x}, t_{1}\right\rangle \cap \sigma_{H}^{\circ}\left\langle\bar{y}, t_{2}\right\rangle \neq \varnothing$ for some $\bar{x}, \bar{y} \in B, t_{1}, t_{2} \in T_{n}(G)$. Then there is an element $g \in G$ such that $g \in \sigma_{H}^{\circ}\left\langle\bar{x}, t_{1}\right\rangle$ and $g \in \sigma_{H}^{\circ}\left\langle\bar{y}, t_{2}\right\rangle$, so, $t_{1}(g[\bar{x}]) \in H$ and $t_{2}(g[\bar{y}]) \in H$. Let $g_{1} \in \sigma_{H}^{\circ}\left\langle\bar{x}, t_{1}\right\rangle$, i.e., $t_{1}\left(g_{1}[\bar{x}]\right) \in H$. 
Thus $t_{1}\left(g_{1}[\bar{x}]\right), t_{1}(g[\bar{x}]), t_{2}(g[\bar{y}]) \in H$, whence, by (18), we obtain $t_{2}\left(g_{1}[\bar{y}]\right) \in H$. Consequently, $g_{1} \in \sigma_{H}^{\circ}\left\langle\bar{y}, t_{2}\right\rangle$, which proves the inclusion $\sigma_{H}^{\circ}\left\langle\bar{x}, t_{1}\right\rangle \subseteq \sigma_{H}^{\circ}\left\langle\bar{y}, t_{2}\right\rangle$. The proof of the reverse inclusion is similar. Thus, $\sigma_{H}^{\circ}\left\langle\bar{x}, t_{1}\right\rangle=\sigma_{H}^{\circ}\left\langle\bar{y}, t_{2}\right\rangle$. This proves (19) and (iii).

$($ iii $) \longrightarrow(i)$ If the premise of (17) is satisfied, then there exists $(\bar{x}, t)$ such that $(\bar{x}, t) \in \sigma_{H}\langle a\rangle \cap \sigma_{H}\langle b\rangle$. Therefore, $t(a[\bar{x}]) \in H$ and $t(b[\bar{x}]) \in H$. Consequently, $a \in \sigma_{H}^{\circ}\langle\bar{x}, t\rangle$ and $b \in \sigma_{H}^{\circ}\langle\bar{x}, t\rangle$. Let $\left(\bar{y}, t_{1}\right) \in \sigma_{H}\langle a\rangle$. Then $t_{1}(a[\bar{y}]) \in H$, whence $a \in \sigma_{H}^{\circ}\left\langle\bar{y}, t_{1}\right\rangle$. Therefore, $\sigma_{H}^{\circ}\langle\bar{x}, t\rangle \cap \sigma_{H}^{\circ}\left\langle\bar{y}, t_{1}\right\rangle \neq \varnothing$, which, by (19), gives $\sigma_{H}^{\circ}\langle\bar{x}, t\rangle=\sigma_{H}^{\circ}\left\langle\bar{y}, t_{1}\right\rangle$. So, $b \in \sigma_{H}^{\circ}\left\langle\bar{y}, t_{1}\right\rangle$, i.e., $t_{1}(b[\bar{y}]) \in H$. Thus, $\left(\bar{y}, t_{1}\right) \in \sigma_{H}\langle b\rangle$ and $\sigma_{H}\langle a\rangle \subseteq \sigma_{H}\langle b\rangle$. Analogously we can prove $\sigma_{H}\langle b\rangle \subseteq \sigma_{H}\langle a\rangle$. Therefore, $\sigma_{H}\langle a\rangle=\sigma_{H}\langle b\rangle$. This proves (17).

Note that according to Theorem 4.4 the empty subset of a Menger algebra is bistrong since for $H=\varnothing$ the premise and the conclusion of (18) are false, so this implication is true.

Proposition 4.5. Every nonempty normal bicomplex $H$ of a Menger algebra $(G, o)$ is a $\mathcal{P}_{H}$-class different from $W^{H}$.

Proof. Let $h_{1}, h_{2} \in H$ and $t\left(h_{1}[\bar{x}]\right) \in H$ for some $t \in T_{n}(G), \bar{x} \in B$. Then, by (3), we have $t\left(h_{2}[\bar{x}]\right) \in H$. Similarly, from $t\left(h_{2}[\bar{x}]\right) \in H$ we obtain $t\left(h_{1}[\bar{x}]\right) \in H$. Thus,

$$
\left(\forall t \in T_{n}(G)\right)(\forall \bar{x} \in B)\left(t\left(h_{1}[\bar{x}]\right) \in H \longleftrightarrow t\left(h_{2}[\bar{x}]\right) \in H\right),
$$

so $\left(h_{1}, h_{2}\right) \in \mathcal{P}_{H}$. Consequently, $H$ is contained in some $\mathcal{P}_{H}$-class. Denote this class by $X$. So, $H \subseteq X$. Now let $g \in X$ and $h \in H$. Then, obviously, $(h, g) \in \mathcal{P}_{H}$, i.e.,

$$
t(h[\bar{x}]) \in H \longleftrightarrow t(g[\bar{x}]) \in H
$$

for all $t \in T_{n}(G), \bar{x} \in B$. From this, for the identity translation $t$ and $\bar{x}=\bar{e}$, we get $h \in H \longleftrightarrow g \in H$. So, $g \in H$ and $X \subseteq H$, whence $H=X$. Since $H \cap W^{H}=\varnothing$, the class $X=H$ is different from $W^{H}$.

Corollary 4.6. Every nonempty bistrong subset $H$ of a Menger algebra $(G, o)$ is an equivalence class of the principal congruence $\mathcal{P}_{H}$ different from biresidue $W^{H}$.

Proof. Every bistrong subset $H \neq \varnothing$ satisfies (18) for any elements $g_{1}, g_{2} \in G$ and $\bar{x}, \bar{y} \in B, t_{1}, t_{2} \in T_{n}(G)$. Replacing $t_{1}$ by the identity translation and $\bar{x}$ by $\bar{e}$ we see that $H$ is a normal bicomplex. Hence, by Proposition 4.5, it is a $\mathcal{P}_{H}$-class different from $W^{H}$.

Proposition 4.7. Let $H$ be a bistrong subset of a Menger algebra $(G, o)$. Then the $\mathcal{P}_{H}$-classes are nonempty members of the family

$$
\mathcal{D}=\left\{\sigma_{H}^{\circ}\langle\bar{x}, t\rangle \mid(\bar{x}, t) \in B \times T_{n}(G)\right\} \cup\left\{W^{H}\right\} .
$$


Proof. First, make sure that the family $\mathcal{D}$ is a partition of the set $G$. In fact, $W^{H} \subseteq G$ and $\sigma_{H}^{\circ}\langle\bar{x}, t\rangle \subseteq G$ for all $t \in T_{n}(G)$ and $\bar{x} \in B$. Thus, $\bigcup \mathcal{D} \subseteq G$. Conversely, for an arbitrary element $g \in G$ we have two possibilities: either $\left(\forall t \in T_{n}(G)\right)(\forall \bar{x} \in B) t(g[\bar{x}]) \notin H$, or $t(g[\bar{x}]) \in H$ for some $t \in T_{n}(G)$ and $\bar{x} \in B$. In the first case $g \in W^{H}$, in the second $g \in \sigma_{H}^{\circ}\langle\bar{x}, t\rangle$ for some $t \in T_{n}(G)$ and $\bar{x} \in B$. Therefore, $g \in \bigcup \mathcal{D}$, so $G \subseteq \bigcup \mathcal{D}$. Thus, $G=\bigcup \mathcal{D}$, i.e., $\mathcal{D}$ covers $G$.

Next, we show that the members of $\mathcal{D}$ are pairwise disjoint. Indeed, if there is $g \in G$ such that $g \in \sigma_{H}^{\circ}\left\langle\bar{x}_{1}, t_{1}\right\rangle \cap W^{H}$, where $t_{1} \in T_{n}(G), \bar{x}_{1} \in B$, then $t_{1}\left(g\left[\bar{x}_{1}\right]\right) \in H$ and $t(g[\bar{x}]) \notin H$ for all $t \in T_{n}(G), \bar{x} \in B$, so $t_{1}\left(g\left[\bar{x}_{1}\right]\right) \in H$ and $t_{1}\left(g\left[\bar{x}_{1}\right]\right) \notin H$ at the same time, which is impossible. Therefore, $\sigma_{H}^{\circ}\left\langle\bar{x}_{1}, t_{1}\right\rangle \cap$ $W^{H}=\varnothing$ for all $t_{1} \in T_{n}(G), \bar{x}_{1} \in B$. If $\sigma_{H}^{\circ}\left\langle\bar{x}_{1}, t_{1}\right\rangle \cap \sigma_{H}^{\circ}\left\langle\bar{x}_{2}, t_{2}\right\rangle \neq \varnothing$, then, according to (19), $\sigma_{H}^{\circ}\left\langle\bar{x}_{1}, t_{1}\right\rangle=\sigma_{H}^{\circ}\left\langle\bar{x}_{2}, t_{2}\right\rangle$. So, if $\sigma_{H}^{\circ}\left\langle\bar{x}_{1}, t_{1}\right\rangle \neq \sigma_{H}^{\circ}\left\langle\bar{x}_{2}, t_{2}\right\rangle$, then $\sigma_{H}^{\circ}\left\langle\bar{x}_{1}, t_{1}\right\rangle \cap \sigma_{H}^{\circ}\left\langle\bar{x}_{2}, t_{2}\right\rangle=\varnothing$. Hence, $\mathcal{D}$ is a partition of $G$.

Now let $(a, b) \in \mathcal{P}_{H}$, i.e., $\sigma_{H}\langle a\rangle=\sigma_{H}\langle b\rangle$. If $\sigma_{H}\langle a\rangle=\varnothing$, then $a, b \in W^{H}$. If $\sigma_{H}\langle a\rangle \neq \varnothing$, then there exist $t \in T_{n}(G)$ and $\bar{x} \in B$ such that $(\bar{x}, t) \in \sigma_{H}\langle a\rangle=$ $\sigma_{H}\langle b\rangle$, so $t(a[\bar{x}]) \in H$ and $t(b[\bar{x}]) \in H$. Hence, $a, b \in \sigma_{H}^{\circ}\langle\bar{x}, t\rangle$. This shows that any $a, b \in G$ such that $(a, b) \in \mathcal{P}_{H}$ belong to the same equivalence class of the relation $\mathcal{P}_{H}$.

Conversely, if $a, b \in \sigma_{H}^{\circ}\langle\bar{x}, t\rangle$ for some $t \in T_{n}(G)$ and $\bar{x} \in B$, then $t(a[\bar{x}]) \in H$ and $t(b[\bar{x}]) \in H$, so $(\bar{x}, t) \in \sigma_{H}\langle a\rangle \cap \sigma_{H}\langle b\rangle$. Consequently, $\sigma_{H}\langle a\rangle \cap \sigma_{H}\langle b\rangle \neq \varnothing$, so $\sigma_{H}\langle a\rangle=\sigma_{H}\langle b\rangle$ because $H$ is bistrong. Thus, $(a, b) \in \mathcal{P}_{H}$. Hence $\sigma_{H}^{\circ}\langle\bar{x}, t\rangle$ is an equivalence class of the relation $\mathcal{P}_{H}$.

To any polynomial $t \in T_{n}(G)$ and any vector $\bar{a} \in B$ we associate the polynomial $t^{\bar{a}} \in T_{n}(G)$ obtained from $t$ by assigning $[\bar{a}]$ to all elements standing in the square brackets except for the variable $x$ and the elements standing directly before the left square bracket. For example, to $t(x)=u\left[w_{1} \ldots w_{i-1} x w_{i+1} \ldots w_{n}\right]$ we associate the polynomial $t^{\bar{a}}(x)=u\left[w_{1}[\bar{a}] \ldots w_{i-1}[\bar{a}] x w_{i+1}[\bar{a}] \ldots w_{n}[\bar{a}]\right]$, to $t_{1}(x)=u\left[\left.\bar{w}\right|_{i} v\left[\left.\bar{s}\right|_{j} x\right]\right]$ we associate $t_{1}^{\bar{a}}(x)=u\left[\left.\bar{w} * \bar{a}\right|_{i} v\left[\left.\bar{s} * \bar{a}\right|_{j} x\right]\right]$.

Using (11) it is easy to show that

$$
t^{\bar{a}}(g[\bar{a}])=t(g)[\bar{a}]
$$

for all $g \in G, \bar{a} \in B, t \in T_{n}(G)$.

Theorem 4.8. Let $H$ be a nonempty bistrong subset of a Menger algebra $(G, o)$. Then each $\mathcal{P}_{H}$-class $X$ different from $W^{H}$ is bistrong, $W^{H} \subseteq W^{X}, \mathcal{P}_{H} \subseteq \mathcal{P}_{X}$. Furthermore, the restrictions of $\mathcal{P}_{H}$ and $\mathcal{P}_{X}$ on $G \backslash W^{X}$ coincide.

Proof. Let $\sigma_{X}\langle a\rangle \cap \sigma_{X}\langle b\rangle \neq \varnothing$, then $(\bar{x}, t) \in \sigma_{X}\langle a\rangle \cap \sigma_{X}\langle b\rangle$ for some $t \in T_{n}(G)$ and $\bar{x} \in B$. Therefore, $t(a[\bar{x}]) \in X$ and $t(b[\bar{x}]) \in X$. Since the $\mathcal{P}_{H}$-class $X$ is different from $W^{H}$, by Proposition 4.7, we have $X=\sigma_{H}^{\circ}\left\langle\bar{y}, t_{1}\right\rangle$ for some $t_{1} \in T_{n}(G), \bar{y} \in B$. Consequently, $t(a[\bar{x}]) \in \sigma_{H}^{\circ}\left\langle\bar{y}, t_{1}\right\rangle$ and $t(b[\bar{x}]) \in \sigma_{H}^{\circ}\left\langle\bar{y}, t_{1}\right\rangle$, whence $t_{1}(t(a[\bar{x}])[\bar{y}]) \in H$ and $t_{1}(t(b[\bar{x}])[\bar{y}]) \in H$. Thus, $t_{1}\left(t^{\bar{y}}(a[\bar{x}][\bar{y}])\right) \in H$ and $t_{1}\left(t^{\bar{y}}(b[\bar{x}][\bar{y}])\right) \in H$, i.e., $t_{1}\left(t^{\bar{y}}(a[\bar{x} * \bar{y}])\right) \in H$ and $t_{1}\left(t^{\bar{y}}(b[\bar{x} * \bar{y}])\right) \in H$. This means that $\left(t_{1} \circ t^{\bar{y}}\right)(a[\bar{x} * \bar{y}]) \in H$ and $\left(t_{1} \circ t^{\bar{y}}\right)(b[\bar{x} * \bar{y}]) \in H$, so $\left(\bar{x} * \bar{y}, t_{1} \circ t^{\bar{y}}\right) \in \sigma_{H}\langle a\rangle$ and $\left(\bar{x} * \bar{y}, t_{1} \circ t^{\bar{y}}\right) \in \sigma_{H}\langle b\rangle$. Thus, $\sigma_{H}\langle a\rangle \cap \sigma_{H}\langle b\rangle \neq \varnothing$. Since $H$ is bistrong, the last 
implies $\sigma_{H}\langle a\rangle=\sigma_{H}\langle b\rangle$. Now let $\left(\bar{z}, t_{2}\right) \in \sigma_{X}\langle a\rangle$, then $t_{2}(a[\bar{z}]) \in X=\sigma_{H}^{\circ}\left\langle\bar{y}, t_{1}\right\rangle$, which gives $t_{1}\left(t_{2}(a[\bar{z}])[\bar{y}]\right) \in H$. Consequently, $\left(t_{1} \circ t_{2}^{\bar{y}}\right)(a[\bar{z} * \bar{y}]) \in H$, whence we obtain $\left(\bar{z} * \bar{y}, t_{1} \circ t_{2}^{\bar{y}}\right) \in \sigma_{H}\langle a\rangle=\sigma_{H}\langle b\rangle$. So, $\left(t_{1} \circ t_{2}^{\bar{y}}\right)(b[\bar{z} * \bar{y}]) \in H$, i.e, $t_{1}\left(t_{2}(b[\bar{z}])[\bar{y}]\right) \in H$. Hence, $t_{2}(b[\bar{z}]) \in \sigma_{H}^{\circ}\left\langle\bar{y}, t_{1}\right\rangle=X$, which means that $\left(\bar{z}, t_{2}\right) \in$ $\sigma_{X}\langle b\rangle$. In this way we have proved the inclusion $\sigma_{X}\langle a\rangle \subseteq \sigma_{X}\langle b\rangle$. The proof of $\sigma_{X}\langle b\rangle \subseteq \sigma_{X}\langle a\rangle$ is similar. Therefore, $\sigma_{X}\langle a\rangle=\sigma_{X}\langle b\rangle$. This proves that $X$ is a bistrong subset of $(G, o)$.

Let $(a, b) \in \mathcal{P}_{H}$, i.e., $\sigma_{H}\langle a\rangle=\sigma_{H}\langle b\rangle$. If $\left(\bar{v}, t_{3}\right) \in \sigma_{X}\langle a\rangle$ for some $t_{3} \in T_{n}(G)$ and $\bar{v} \in B$, then $t_{3}(a[\bar{v}]) \in X$. So, $t_{3}(a[\bar{v}]) \in \sigma_{H}^{\circ}\left\langle\bar{y}, t_{1}\right\rangle$, whence we obtain $t_{1}\left(t_{3}(a[\bar{v}])[\bar{y}]\right) \in H$. Thus, $t_{1}\left(t_{3}^{\bar{y}}(a[\bar{v}][\bar{y}])\right) \in H$, which gives $\left(t_{1} \circ t_{3}^{\bar{y}}\right)(a[\bar{v} * \bar{y}]) \in H$. So, $\left(\bar{v} * \bar{y}, t_{1} \circ t_{3}^{\bar{y}}\right) \in \sigma_{H}\langle a\rangle=\sigma_{H}\langle b\rangle$. Consequently, $\left(\bar{v}, t_{3}\right) \in \sigma_{X}\langle b\rangle$ and $\sigma_{X}\langle a\rangle \subseteq$ $\sigma_{X}\langle b\rangle$. Analogously we show the reverse inclusion. Therefore, $\sigma_{X}\langle a\rangle=\sigma_{X}\langle b\rangle$ and $(a, b) \in \mathcal{P}_{X}$. Hence $\mathcal{P}_{H} \subseteq \mathcal{P}_{X}$.

Thus, $W^{H}$ is contained in some $\mathcal{P}_{X}$-class. Let $a \in W^{H}$, i.e., $\sigma_{H}\langle a\rangle=\varnothing$. Suppose that $\sigma_{X}\langle a\rangle \neq \varnothing$. Then $t_{4}(a[\bar{w}]) \in X$ for some $t_{4} \in T_{n}(G)$ and $\bar{w} \in B$. Since $X=\sigma_{H}^{\circ}\left\langle\bar{y}, t_{1}\right\rangle$, the above gives $t_{4}(a[\bar{w}]) \in \sigma_{H}^{\circ}\left\langle\bar{y}, t_{1}\right\rangle$, so $t_{1}\left(t_{4}(a[\bar{w}])[\bar{y}]\right) \in H$, therefore $\left(\bar{w} * \bar{y}, t_{1} \circ t_{4}^{\bar{y}}\right) \in \sigma_{H}\langle a\rangle$, whence $\sigma_{H}\langle a\rangle \neq \varnothing$, i.e., $a \notin W^{H}$, which is impossible, since $a \in W^{H}$. Thus, our supposition is wrong. Therefore $\sigma_{X}\langle a\rangle=$ $\varnothing$, i.e., $a \in W^{X}$. Hence $W^{H} \subseteq W^{X}$.

Since $\mathcal{P}_{H} \subseteq \mathcal{P}_{X}$, the set $W^{X}$ is a union of $\mathcal{P}_{H}$-classes. We show that the restrictions of $\mathcal{P}_{H}$ and $\mathcal{P}_{X}$ on $G \backslash W^{X}$ coincide. For this enough to show that for any $a, b \in G \backslash W^{X}$ such that $(a, b) \in \mathcal{P}_{X}$ we have $(a, b) \in \mathcal{P}_{H}$. In fact, if $a, b \in G \backslash W^{X}$ and $(a, b) \in \mathcal{P}_{X}$, then $\sigma_{X}\langle a\rangle=\sigma_{X}\langle b\rangle \neq \varnothing$. Thus, there are $t_{5} \in T_{n}(G)$ and $\bar{u} \in B$ such that $t_{5}(a[\bar{u}]) \in X=\sigma_{H}^{\circ}\left\langle\bar{y}, t_{1}\right\rangle$ and $t_{5}(b[\bar{u}]) \in X=\sigma_{H}^{\circ}\left\langle\bar{y}, t_{1}\right\rangle$. Hence $t_{1}\left(t_{5}(a[\bar{u}])[\bar{y}]\right) \in H$ and $t_{1}\left(t_{5}(b[\bar{u}])[\bar{y}]\right) \in H$, so, $\left(\bar{u} * \bar{y}, t_{1} \circ t_{5}^{\bar{y}}\right) \in \sigma_{H}\langle a\rangle$ and $\left(\bar{u} * \bar{y}, t_{1} \circ t_{5}^{\bar{y}}\right) \in \sigma_{H}\langle b\rangle$. Consequently, $\sigma_{H}\langle a\rangle \cap \sigma_{H}\langle b\rangle \neq \varnothing$, which implies $\sigma_{H}\langle a\rangle=\sigma_{H}\langle b\rangle$. Therefore, $(a, b) \in \mathcal{P}_{H}$. This completes our proof.

Corollary 4.9. Let $H$ be a bistrong subset of a Menger algebra $(G, o)$. Then for any translation $t \in T_{n}(G)$ and every $\bar{x} \in B$ the set $\sigma_{H}^{\circ}\langle\bar{x}, t\rangle$ is bistrong.

Proof. It was noted above that the empty subset is bistrong, so if $\sigma_{H}^{\circ}\langle\bar{x}, t\rangle=\varnothing$ for some $t \in T_{n}(G)$ and $\bar{x} \in B$, then, obviously, $\sigma_{H}^{\circ}\langle\bar{x}, t\rangle$ is bistrong. If $\sigma_{H}^{\circ}\langle\bar{x}, t\rangle \neq$ $\varnothing$, then, by Proposition 4.7, $\sigma_{H}^{\circ}\langle\bar{x}, t\rangle$ is a $\mathcal{P}_{H}$-class different from $W^{H}$. Therefore, by Theorem 4.8, $\sigma_{H}^{\circ}\langle\bar{x}, t\rangle$ is bistrong.

Proposition 4.10. Each $\varepsilon$-class $X$ of an lv-cancellative congruence $\varepsilon$ on a Menger algebra $(G, o)$ is bistrong and $\varepsilon \subseteq \mathcal{P}_{X}$. Moreover, the relations $\varepsilon$ and $\mathcal{P}_{X}$ coincide on $G \backslash W^{X}$.

Proof. Let $\sigma_{X}\langle a\rangle \cap \sigma_{X}\langle b\rangle \neq \varnothing$, then $(\bar{x}, t) \in \sigma_{X}\langle a\rangle$ and $(\bar{x}, t) \in \sigma_{X}\langle b\rangle$ for some $t \in$ $T_{n}(G)$ and $\bar{x} \in B$. Thus, $t(a[\bar{x}]) \in X$ and $t(b[\bar{x}]) \in X$, whence $(t(a[\bar{x}]), t(b[\bar{x}])) \in$ $\varepsilon$. Since $\varepsilon$ is a $v$-cancellative congruence, the last implies $(a[\bar{x}], b[\bar{x}]) \in \varepsilon$, which, by $l$-cancellativity, gives $(a, b) \in \varepsilon$. But $\varepsilon \subseteq \mathcal{P}_{X}$ (Proposition 4.2), so, $(a, b) \in$ $\mathcal{P}_{X}$, i.e., $\sigma_{X}\langle a\rangle=\sigma_{X}\langle b\rangle$. This shows that $X$ is a bistrong subset of $(G, o)$. 
Moreover, from $\varepsilon \subseteq \mathcal{P}_{X}$ we obtain

$$
\varepsilon \cap\left(G \backslash W^{X}\right) \times\left(G \backslash W^{X}\right) \subseteq \mathcal{P}_{X} \cap\left(G \backslash W^{X}\right) \times\left(G \backslash W^{X}\right) .
$$

On the other hand, if $(a, b) \in \mathcal{P}_{X} \cap\left(G \backslash W^{X}\right) \times\left(G \backslash W^{X}\right)$, then $a, b \in G \backslash W^{X}$ and

$$
t(a[\bar{x}]) \in X \longleftrightarrow t(b[\bar{x}]) \in X
$$

for all $t \in T_{n}(G), \bar{x} \in B$. Since for any $a \in G \mid W^{X}$ there are $t_{1} \in T_{n}(G)$ and $\bar{y} \in B$ such that $t_{1}(a[\bar{y}]) \in X$, we have $t_{1}(b[\bar{y}]) \in X$. Therefore, $\left(t_{1}(a[\bar{y}]), t_{1}(b[\bar{y}])\right) \in \varepsilon$, whence $(a, b) \in \varepsilon$ because the relation $\varepsilon$ is $l v$-cancellative. So,

$$
\mathcal{P}_{X} \cap\left(G \backslash W^{X}\right) \times\left(G \backslash W^{X}\right) \subseteq \varepsilon \cap\left(G \backslash W^{X}\right) \times\left(G \backslash W^{X}\right) .
$$

From these two inclusions we obtain

$$
\varepsilon \cap\left(G \backslash W^{X}\right) \times\left(G \backslash W^{X}\right)=\mathcal{P}_{X} \cap\left(G \backslash W^{X}\right) \times\left(G \backslash W^{X}\right) .
$$

Thus, the relations $\varepsilon$ and $\mathcal{P}_{X}$ coincide on the set $G \backslash W^{X}$.

\section{References}

[1] Clifford A. H., Preston G. B., The algebraic theory of semigroups, Amer. Math. Soc., Providence, R. I., vol. 1, 1964; vol. 2, 1967.

[2] Dubreil P., Contribution á la théorie des demi-groupes, Mém. Acad. Sci. Inst. France 63, no.3 (1941), 1-52.

[3] Dudek W. A., Trokhimenko V. S. Algebras of multiplace functions, Walter de Gruyter GmbH \& Co. KG, Berlin/Boston, 2012.

[4] Schein B.M., Trohimenko V.S. Algebras of multiplace functions, Semigroup Forum 17 (1979), 1-64.

[5] Schein B.M. Embedding semigroups in inverse semigroups, Transl., Ser. 2. Am. Math. Soc. 139 (1988), 93-116 (translation from Mat. Sb., Nov. Ser. 55(97) (1961), 379-400).

Dudek W. A.

Institute of Mathematics and Computer Science

Wroclaw University of Technology 50-370 Wroclaw

Poland

Email: Wieslaw.Dudek@im.pwr.wroc.pl
Trokhimenko V. S.

Department of Mathematics Pedagogical University 21100 Vinnitsa

Ukraine

Email: vtrokhim@gmail.com 